\title{
The effect of transverse electric fields on dielectric, piezoelectric, elastic and thermal properties of the Rochelle salt $\mathrm{NaKC}_{4} \mathrm{H}_{4} \mathrm{O}_{6} \cdot 4 \mathrm{H}_{2} \mathrm{O}$
}

\author{
R.R.Levitskii ${ }^{1}$, I.R.Zachek ${ }^{2}$, A.S.Vdovych ${ }^{1}$, I.V.Stasyuk ${ }^{1}$ \\ ${ }^{1}$ Institute for Condensed Matter Physics of the National Academy of Sciences of Ukraine, \\ 1 Svientsitskii Str., 79011 Lviv, Ukraine \\ 2 Lviv Polytechnic National University, 12 Bandera Str., 79013 Lviv, Ukraine
}

Received May 26, 2009

\begin{abstract}
Modified four-sublattice model for Rochelle salt by taking into account piezoelectric interactions with shear strain $\varepsilon_{4}, \varepsilon_{5}$ and $\varepsilon_{6}$ is proposed. Components of polarization vector and static dielectric permittivity tensor for both mechanically clamped and free crystals, their piezoelectric characteristics and elastic modules are derived in the mean field approximation. A comprehensive study of transverse field effect on phase transition temperatures, dielectric and elastic properties of Rochelle salt has been performed for the first time.
\end{abstract}

Key words: ferroelectrics, rochelle salt, piezoelectric modules

PACS: 77.84.-s, 64.60.Cn, 77.22.-d, 77.80.-e, 77.80.Bh, 77.65.Bn

\section{Introduction}

Rochelle salt (Rs) is a special ferroelectric hydrogen bonded compound. Some details of its structure and a precise mechanism of the phase transitions are still unclear. The crystal structure of Rochelle salt and its properties are described in [1,2]. The most peculiar to Rochelle salt is the presence of two Curie points. The phase transitions are of the second order. The ferroelectric phase, existing in the temperature range between 255 and $297 \mathrm{~K}$, is monoclinic and belongs to the space group $C_{2}^{2}-P 2_{1}$. The spontaneous polarization is directed along the $a$ axis. In the lowtemperature and high-temperature paraelectric phases the crystal has an orthorhombic space group $D_{2}^{3}-P 2_{1} 2_{1} 2_{1}$. The unit cell contains four formula units.

Structural studies do not completely clarify the microscopic origin of the phase transitions in Rochelle salt. The microwave dielectric relaxation and a critical slowing down near the transition points indicate the order-disorder type phase transition [5]. On the contrary, the soft mode observed in infrared reflectivity spectra, by Raman scattering in the low-temperature paraelectric phase [6], and by microwave dielectric measurements [7] indicates the phase transition of a displacement type. The soft mode in the paraelectric phase is related to the changes in the crystal structure (displacements of the oxygen atoms $\mathrm{O}(8)$ along the axis $a$ and rotation of the water molecules bonded to ions $\mathrm{O}(9)$ and $\mathrm{O}(10)$ ) taking place at the transition to the ferroelectric phase [8]. This picture is also confirmed by inelastic neutron scattering data [9]. The static displacements produce additional dipole moments of the structural elements of Rochelle salt at the ferroelectric phase transitions. Such displacements can be also treated as changes in the ratio of populations within double positions in the disordered paraelectric structure, revealed in $[10,11]$, whereas large values of anisotropic structure factors can be related to the local disorder [12]. The existence of double atomic positions was explored within the so-called split-atom model for Rochelle salt [13].

The order-disorder scenario for phase transitions in Rochelle salt underlies the half-microscopic Mitsui model [14] that takes into account the two key effects: asymmetry of population of two local atom positions and compentation of the electric dipole moments in paraelectric phases. Despite a simplified approach (two sublattices only), the Mitsui model even in the mean field approximation 
permits, at a proper choice of the theory parameters, to explain the existence of two Curie points in Rochelle salt and to describe the behavior of its dielectric characteristics and heat capacity. Later [5,15] the Mitsui model was formulated in terms of pseudospin operators. In [15-17] the thermodynamic characteristics of Mitsui model were calculated in the mean field approximation. In $[16,17]$ tunneling of the ordering units was taken into account. Relaxational phenomena in ferroelectrics described by the Mitsui model were explored in $[15,18]$. In [15] using the stochastic Glauber [19] model and in [18] using the Bloch equation method [20] the relaxation times for deuterated and pure Rochelle salt were calculated.

The main purpose of the above mentioned works was to determine whether the Mitsui model was capable of describing the experimental data for some selected physical properties of Rochelle salt. The other characteristics were not always calculated with the account of the obtained values of the theory parameters. Therefore, it was not firmly established whether the Mitsui model is applicable to Rochelle salt or not. Also, in [16] a necessity of a thorough study of possible phase transitions in the Mitsui model was indicated, and a very approximate phase transition of the model without tunneling was constructed. Later, the phase diagrams for the Mitsui model (also with tunneling) were more thoroughly studied in [21,22]. The complete phase diagram of the Mitsui model, also with tunneling, was constructed only in [23]. In [24-26] the thermodynamic and (using the stochastic Glauber model [19]) dynamic characteristics of pure and deuterated Rochelle salt were calculated, and the values of the theory parameters providing a fair description of the available experimental data were obtained for several physical characteristics of the crystals.

Crystals of Rochelle salt are non-centrosymmetric and piezoelectric in paraelectric and ferroelectric phases. This fact essentially affects their physical properties. Prior to work [31], the model consideration of dielectric response in Rochelle salt had been restricted to the static limit and to the microwave region. Qualitatively correct results for high-frequency characteristics can be obtained only within a model that does take into account the piezoelectric coupling. The conventional Mitsui model does not distinguish free and clamped crystals in the static limit and is not capable of reproducing the effect of crystal clamping by high-frequency electric field. It permits to calculate [24-26] the dielectric permittivity and relaxation times of a free crystal only. It was obtained, however, that the relaxation time, exhibiting a critical slowing down at the Curie points, actually diverges at these points, whereas experiments [5] indicate that it should be large but remain finite. Also, the calculated permittivity has a sharp minimum at all frequencies which qualitatively differs from the experimentally observed behavior.

A proposed $[27,28]$ modification of the Mitsui model takes into account the piezoelectric coupling with the shear strain $\varepsilon_{4}$. It allows one to calculate the piezoelectric and elastic characteristics of Rochelle salt as well as susceptibilities of both free and clamped crystals and to obtain a correct temperature behavior of the relaxation times and longitudinal dynamic permittivity near the Curie points.

One should also mention a modification of the phenomenological Landau theory [29] for the systems with a double critical point that describes the properties of Rochelle salt in a wide temperature, pressure, and composition (when $\mathrm{K}$ is replaced by $\mathrm{NH}_{4}$ ) ranges. Also, in [30] the thermodynamic, longitudinal dielectric, piezoelectric, and elastic characteristics of the disordered modified Mitsui model were calculated. A thorough analysis of the obtained results was performed; possible changes in the physical characteristics of Rochelle salt with deuterated were discussed.

In [31], within the framework of the modified Mitsui model, a dynamic dielectric response of Rochelle salt was considered, with taking into account the dynamics of the piezoelectric strain. The phenomena of crystal clamping by high-frequency electric field, piezoelectric resonance, and microwave dispersion were described. Ultrasound velocity and attenuation were calculated, and peculiarities of their temperature dependence at the Curie points were described. The existence of a cutoff frequency in the frequency dependence of attenuation was shown.

Within the model proposed in [27], the effect of the shear stress $\sigma_{4}$ [32], longitudinal electric field [33], and hydrostatic pressure [34] on the physical characteristics of Rochelle salt was explored. A satisfactory agreement with experimental data was obtained for the field effect on the static dielectric permittivity near the lower Curie temperature. At the upper Curie temperature, 
an effective field should be used in calculations to describe the experiment. A possible role of space-charge build-up in the screening of the external field at high temperatures is discussed. The effect of the longitudinal electric field on the dynamic dielectric, elastic, piezoelectric, and acoustic characteristics of Rochelle salt was studied. It was shown that the temperature behavior of the permittivity in the resonance region can be qualitatively changed by the external field; this is due to the increase of the resonance frequencies with the field. It was also established that the longitudinal field strongly increases the cutoff frequency, as well as the microwave dielectric relaxation frequency. These effects are due to the decrease of the relaxation time with the field.

In [34], the effect of hydrostatic pressure on the physical characteristics of Rochelle salt is explored. The available experimental data and dependences of temperature and pressure variation of the physical characteristics on the theory parameters are analysed. An optimal set of the model parameters is obtained, allowing one to calculate the temperature dependences of dielectric, elastic, piezoelectric, and thermal characteristics at different pressures and to describe the available experimental data.

In [35], within the framework of the modified Mitsui model, the effect of tunnelling on thermodynamic, dielectric, piezoelectric, and elastic characteristics of Rochelle salt were explored. It was shown that tunneling hardly affects the calculated quantities, but improves an agreement between the theory and experiment for spontaneous polarization. It would be interesting to study the effect of tunneling on the dynamic characteristics of Rochelle salt within this model. Important is a structural investigation of Rochelle salt crystals, aimed at answering the question whether tunneling of the ordering units takes place here indeed.

Hence, the modified Mitsui model [27] made possible a proper description of longitudinal dielectric, piezoelectric, and elastic characteristics of Rochelle salt and its behavior in the presence of longitudinal electric field and hydrostatic pressure. However, this model oversimplifies the real structure of the crystal, postulating the direction of the ferroelectric axis among the three possible second order axes. As a result, the approach becomes essentially "one-dimensional" and does not permit a complete description of all dielectric, piezoelectric, and elastic characteristics of the crystal. A possible generalization of the Mitsui model by its transformation to a "three-dimensional" model that takes into account all four translationally non-equivalent groups of atoms in a unit cell was proposed in [36]. Within the framework of the order-disorder scenario, the equilibrium positions of non-equivalent atomic groups are simulated by a four-sublattice pseudospin model that allows one too calculate all components of the tensors of the crystal characteristics, and to explore the effects of transverse (perpendicular to the ferroelectric axis $a$ ) electric fields. Within the mean field approximation it has been shown that the transverse electric field $E_{y}$ partially suppresses the spontaneous polarization and narrows the temperature range of the ferroelectric phase (this roughly corresponds to the experimentally observed effect [37]), and gives rise to the jumps of the transverse dielectric permittivity at the transition points, the jump magnitude being proportional to $E_{y}^{2}$.

If properly generalized, the model proposed in [36] can be used in order to calculate the components of the tensors of static and dynamic dielectric permittivities and components of the tensors of piezoelectric and elastic coefficients of Rochelle salt, and their behavior in the presence of transverse and longitudinal electric fields.

Therefore, in this paper we propose a modified four-sublattice pseudospin model for Rochelle salt, where the piezoelectric coupling with the shear strains $\varepsilon_{4}, \varepsilon_{5}$, and $\varepsilon_{6}$ are taken into account. In the mean field approximation, the thermodynamic and transverse dielectric, piezoelectric, and elastic characteristics of Rochelle salt are calculated. The effect of the transverse electric field on these characteristics is explored.

\section{A four-sublattice model: the Hamiltonian}

In order to describe the phase transitions in Rochelle salt and its thermodynamic characteristics we use the "three-dimensional" model [36] that takes into account the presence of four translationally non-equivalent groups of atoms in a unit cell, whose positions are related by elements 
of the paraelectric point group [3,4]. These structural units are non-centrosymmetric. We assign them dipole moments $\mathbf{d}_{q f}(f=1, \ldots, 4)$. In the paraelectric phase, the sum of these moments is zero. Changes $\Delta \mathbf{d}_{q f}$ in these dipole moments are responsible for the appearance of spontaneous polarization in the ferroelectric state. The vectors $\mathbf{d}_{q f}$ are oriented at certain angles to the crystallographic axes and have both longitudinal and transverse components with respect to the axis $a$ (figure 1).
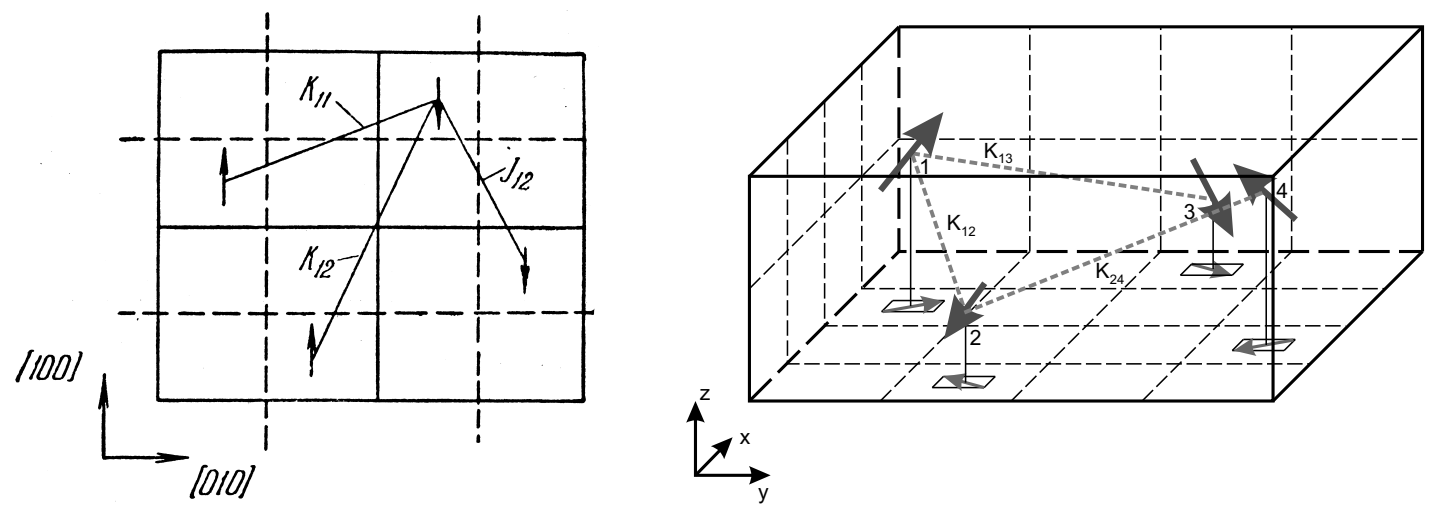

Figure 1. Orientations of dipole moments creating the net polarization in a primitive cell of Rochelle salt crystals: conventional Mitsui model (left) and the proposed model (right). In the paraelectric phase all absolute values of pseudospins are equal in all sublattices.

The pseudospin variables $\frac{\sigma_{q 1}}{2}, \ldots, \frac{\sigma_{q 4}}{2}$ describe the changes associated with reordering of dipole moments of structural units: $\mathbf{d}_{q f}=\mu_{f} \frac{\sigma_{q f}}{2}$. The mean values $\left\langle\frac{\sigma}{2}\right\rangle=\frac{1}{2}\left(n_{a}-n_{6}\right)$ are related to the differences in populations of the positions in a two-minima representation of the vectors $\mathbf{d}_{q f}$, whose orientation in the paraelectric phase is shown in figure 1 (right).

In pseudospin representation, the model Hamiltonian, being a generalization of the proposed in [36] Hamiltonian with the piezoelectric coupling and the lattice "seed" part taken into account is, at the same time, a generalization of the [27] Hamiltonian to the "three-dimensional" model

$$
\begin{aligned}
\hat{H}= & \frac{N}{2} v c_{44}^{E 0} \varepsilon_{4}^{2}+\frac{N}{2} v c_{55}^{E 0} \varepsilon_{5}^{2}+\frac{N}{2} v c_{66}^{E 0} \varepsilon_{6}^{2}--N v e_{14}^{0} \varepsilon_{4} E_{1}-N v e_{25}^{0} \varepsilon_{5} E_{2}-N v e_{36}^{0} \varepsilon_{6} E_{3} \\
& -\frac{N}{2} v \chi_{11}^{\varepsilon 0} E_{1}^{2}-\frac{N}{2} v \chi_{22}^{\varepsilon 0} E_{2}^{2}-\frac{N}{2} v \chi_{33}^{\varepsilon 0} E_{3}^{2}-\frac{1}{2} \sum_{q q^{\prime}} \sum_{f=1}^{4} J_{f f}\left(q q^{\prime}\right) \frac{\sigma_{q f}}{2} \frac{\sigma_{q^{\prime} f}}{2} \\
& -\frac{1}{2} \sum_{q q^{\prime}} \sum_{f \neq f^{\prime}} K_{f f^{\prime}}\left(q q^{\prime}\right) \frac{\sigma_{q f}}{2} \frac{\sigma_{q^{\prime} f^{\prime}}}{2}-\Delta \sum_{q}\left(\frac{\sigma_{q 1}}{2}+\frac{\sigma_{q 2}}{2}-\frac{\sigma_{q 3}}{2}-\frac{\sigma_{q 4}}{2}\right) \\
& -\left(\mu_{1} E_{1}-2 \psi_{4} \varepsilon_{4}\right) \sum_{q}\left(\frac{\sigma_{q 1}}{2}+\frac{\sigma_{q 2}}{2}+\frac{\sigma_{q 3}}{2}+\frac{\sigma_{q 4}}{2}\right) \\
& -\left(\mu_{2} E_{2}-2 \psi_{5} \varepsilon_{5}\right) \sum_{q}\left(\frac{\sigma_{q 1}}{2}-\frac{\sigma_{q 2}}{2}-\frac{\sigma_{q 3}}{2}+\frac{\sigma_{q 4}}{2}\right) \\
& -\left(\mu_{3} E_{3}-2 \psi_{6} \varepsilon_{6}\right) \sum_{q}\left(\frac{\sigma_{q 1}}{2}-\frac{\sigma_{q 2}}{2}+\frac{\sigma_{q 3}}{2}-\frac{\sigma_{q 4}}{2}\right)
\end{aligned}
$$

The first nine terms in (2.1) correspond to the seed part of the Hamiltonian, which is independent of the quasispin subsystem and corresponds to the lattice. The "seed" energy includes the elastic, piezoelectric, and dielectric parts, expressed in terms of the electric fields $E_{i}(i=1,2,3)$ and strains $\varepsilon_{j}(j=1+3) . c_{j j}^{E 0}, e_{i j}^{0}, \chi_{i i}^{\varepsilon 0}$ are the "seed" elastic constants, coefficients of piezoelectric stress, and dielectric susceptibilities, $N$ is the number of primitive cells, $v=\bar{v} k_{\mathrm{B}}$ is the primitive cell volume, $k_{\mathrm{B}}$ is the Boltzmann constant. In $(2.1) J_{f f}\left(q q^{\prime}\right)$ and $K_{f f^{\prime}}\left(q q^{\prime}\right)$ are constants of interactions in the same and in different sublattices, respectively. The internal field $\Delta$ describes the 
asymmetry of populations of two positions. The last three terms in (2.1) describe the coupling of the quasispin system with components of external electric field $E_{i}$ and molecular fields induced by piezoelectric coupling linear over strains $\varepsilon_{j}, \mu_{i}$ are the effective dipole moments per one quasispin; $\psi_{j}$ are the deformation potentials. In (2.1) $\sigma_{q f}$ is a quasispin, whose eigenvalues $\sigma_{q f}= \pm 1$ correspond to an ionic group being in a certain minimum of the double asymmetric potential well in the $f$-th sublattices in a cell with the position vector $\mathbf{R}_{q}$.

Performing an identity transformation

$$
\sigma_{q f}=\eta_{f}+\left(\sigma_{q f}-\eta_{f}\right), \quad(f=1,2,3,4),
$$

neglecting quadratic fluctuations and taking into account the symmetry of interaction constants, we present the system Hamiltonian in the mean field approximation in the form

$$
\hat{H}=U+\hat{H}_{s},
$$

where

$$
\begin{aligned}
U= & \frac{N}{2} v c_{44}^{E 0} \varepsilon_{4}^{2}+\frac{N}{2} v c_{55}^{E 0} \varepsilon_{5}^{2}+\frac{N}{2} v c_{66}^{E 0} \varepsilon_{6}^{2}-N v e_{14}^{0} \varepsilon_{4} E_{1}-N v e_{25}^{0} \varepsilon_{5} E_{2}-N v e_{36}^{0} \varepsilon_{6} E_{3} \\
& -\frac{N}{2} v \chi_{11}^{\varepsilon 0} E_{1}^{2}-\frac{N}{2} v \chi_{22}^{\varepsilon 0} E_{2}^{2}-\frac{N}{2} v \chi_{33}^{\varepsilon 0} E_{3}^{2}+\frac{1}{8} J\left(\eta_{1}^{2}+\eta_{2}^{2}+\eta_{3}^{2}+\eta_{4}^{2}\right)+\frac{1}{4} K_{12}\left(\eta_{1} \eta_{2}+\eta_{3} \eta_{4}\right) \\
& +\frac{1}{4} K_{13}\left(\eta_{1} \eta_{3}+\eta_{2} \eta_{4}\right)+\frac{1}{4} K_{14}\left(\eta_{1} \eta_{4}+\eta_{2} \eta_{3}\right) \\
\hat{H}_{s}= & -\sum_{q}\left(\mathcal{H}_{1} \frac{\sigma_{q 1}}{2}+\mathcal{H}_{2} \frac{\sigma_{q 2}}{2}+\mathcal{H}_{3} \frac{\sigma_{q 3}}{2}+\mathcal{H}_{4} \frac{\sigma_{q 4}}{2}\right)
\end{aligned}
$$

In (2.5) the following notations are used

$$
\begin{aligned}
\mathcal{H}_{1} & =\frac{J}{2} \eta_{1}+\frac{K_{12}}{2} \eta_{2}+\frac{K_{13}}{2} \eta_{3}+\frac{K_{14}}{2} \eta_{4}+\Delta-2 \psi_{4} \varepsilon_{4}-2 \psi_{5} \varepsilon_{5}-2 \psi_{6} \varepsilon_{6}+\mu_{1} E_{1}+\mu_{2} E_{2}+\mu_{3} E_{3}, \\
\mathcal{H}_{2} & =\frac{J}{2} \eta_{2}+\frac{K_{12}}{2} \eta_{1}+\frac{K_{13}}{2} \eta_{4}+\frac{K_{14}}{2} \eta_{3}+\Delta-2 \psi_{4} \varepsilon_{4}+2 \psi_{5} \varepsilon_{5}+2 \psi_{6} \varepsilon_{6}+\mu_{1} E_{1}-\mu_{2} E_{2}-\mu_{3} E_{3}, \\
\mathcal{H}_{3} & =\frac{J}{2} \eta_{3}+\frac{K_{12}}{2} \eta_{4}+\frac{K_{13}}{2} \eta_{1}+\frac{K_{14}}{2} \eta_{2}-\Delta-2 \psi_{4} \varepsilon_{4}+2 \psi_{5} \varepsilon_{5}-2 \psi_{6} \varepsilon_{6}-\mu_{1} E_{1}-\mu_{2} E_{2}+\mu_{3} E_{3}, \\
\mathcal{H}_{4} & =\frac{J}{2} \eta_{4}+\frac{K_{12}}{2} \eta_{3}+\frac{K_{13}}{2} \eta_{4}+\frac{K_{14}}{2} \eta_{1}-\Delta-2 \psi_{4} \varepsilon_{4}-2 \psi_{5} \varepsilon_{5}+2 \psi_{6} \varepsilon_{6}+\mu_{1} E_{1}+\mu_{2} E_{2}-\mu_{3} E_{3} .
\end{aligned}
$$

Hence, the mean values of quasispins

$$
\eta_{f}=\operatorname{th} \frac{\beta}{2} \mathcal{H}_{f}
$$

Let us introduce new variables

$$
\begin{aligned}
\xi_{1}=\frac{1}{4}\left(\eta_{1}+\eta_{2}+\eta_{3}+\eta_{4}\right) & =\frac{1}{4}\left(\operatorname{th} \frac{\beta}{2} \mathcal{H}_{1}+\operatorname{th} \frac{\beta}{2} \mathcal{H}_{2}+\operatorname{th} \frac{\beta}{2} \mathcal{H}_{3}+\operatorname{th} \frac{\beta}{2} \mathcal{H}_{4}\right) \\
\xi_{2}=\frac{1}{4}\left(\eta_{1}-\eta_{2}-\eta_{3}+\eta_{4}\right) & =\frac{1}{4}\left(\operatorname{th} \frac{\beta}{2} \mathcal{H}_{1}-\operatorname{th} \frac{\beta}{2} \mathcal{H}_{2}-\operatorname{th} \frac{\beta}{2} \mathcal{H}_{3}+\operatorname{th} \frac{\beta}{2} \mathcal{H}_{4}\right) \\
\xi_{3}=\frac{1}{4}\left(\eta_{1}-\eta_{2}+\eta_{3}-\eta_{4}\right) & =\frac{1}{4}\left(\operatorname{th} \frac{\beta}{2} \mathcal{H}_{1}-\operatorname{th} \frac{\beta}{2} \mathcal{H}_{2}+\operatorname{th} \frac{\beta}{2} \mathcal{H}_{3}-\operatorname{th} \frac{\beta}{2} \mathcal{H}_{4}\right) \\
\sigma & =\frac{1}{4}\left(\eta_{1}+\eta_{2}-\eta_{3}-\eta_{4}\right)=\frac{1}{4}\left(\operatorname{th} \frac{\beta}{2} \mathcal{H}_{1}+\operatorname{th} \frac{\beta}{2} \mathcal{H}_{2}-\operatorname{th} \frac{\beta}{2} \mathcal{H}_{3}-\operatorname{th} \frac{\beta}{2} \mathcal{H}_{4}\right)
\end{aligned}
$$

where the self-consistent fields $\mathcal{H}_{f}$ are given by expressions

$$
\begin{array}{ll}
\mathcal{H}_{1}=\frac{1}{\beta}\left(\gamma_{1}+\gamma_{2}+\gamma_{3}+\delta\right), & \mathcal{H}_{2}=\frac{1}{\beta}\left(\gamma_{1}-\gamma_{2}-\gamma_{3}+\delta\right), \\
\mathcal{H}_{3}=\frac{1}{\beta}\left(\gamma_{1}-\gamma_{2}+\gamma_{3}-\delta\right), & \mathcal{H}_{4}=\frac{1}{\beta}\left(\gamma_{1}+\gamma_{2}-\gamma_{3}-\delta\right),
\end{array}
$$


and

$$
\begin{array}{ll}
\gamma_{1}=\beta\left(\frac{J_{1}}{2} \xi_{1}-2 \psi_{4} \varepsilon_{4}+\mu_{1} E_{1}\right), & \gamma_{2}=\beta\left(\frac{J_{2}}{2} \xi_{2}-2 \psi_{5} \varepsilon_{5}+\mu_{2} E_{2}\right), \\
\gamma_{3}=\beta\left(\frac{J_{3}}{2} \xi_{3}-2 \psi_{6} \varepsilon_{6}+\mu_{3} E_{3}\right), & \delta=\beta\left(\frac{J_{4}}{2} \sigma+\Delta\right)
\end{array}
$$

and

$$
\begin{array}{ll}
J_{1}=J+K_{12}+K_{13}+K_{14}, & J_{2}=J-K_{12}-K_{13}+K_{14}, \\
J_{3}=J-K_{12}+K_{13}-K_{14}, & J_{4}=J+K_{12}-K_{13}-K_{14} .
\end{array}
$$

Parameters $\xi_{1}, \xi_{2}$, and $\xi_{3}$ describe the dipole ordering along the $a, b$, and $c$-axes, respectively, and the parameter $\sigma$ describes the quasispin ordering in the paraelectric phases.

In the paraelectric phases at $E_{i}=0$ and $\sigma_{j}=0$ the mean values of quasispins are $\eta_{1}=\eta_{2}=$ $-\eta_{3}=-\eta_{4}=\eta$ and, respectively, $\xi_{1 p}=\xi_{2 p}=\xi_{3 p}=0$,

$$
\sigma_{p}=\operatorname{th} \frac{\beta}{2}\left(\frac{J_{4}}{2} \sigma_{p}+\Delta\right) .
$$

If $E_{2} \neq 0$ and $\sigma_{5} \neq 0$, then $\eta_{1}=-\eta_{3}=\eta_{13}, \eta_{2}=-\eta_{4}=\eta_{24}$ and $\xi_{1 p}(2)=\xi_{3 p}(2)=0$,

$$
\begin{aligned}
\xi_{2 p}(2)= & \frac{1}{2}\left[\operatorname{th} \frac{\beta}{2}\left(\frac{J_{2}}{2} \xi_{2 p}(2)-2 \psi_{5} \varepsilon_{5}+\mu_{2} E_{2}+\frac{J_{4}}{2} \sigma_{p}(2)+\Delta\right)\right. \\
& \left.+\operatorname{th} \frac{\beta}{2}\left(\frac{J_{2}}{2} \xi_{2 p}(2)-2 \psi_{5} \varepsilon_{5}+\mu_{2} E_{2}-\frac{J_{4}}{2} \sigma_{p}(2)-\Delta\right)\right], \\
\sigma_{p}(2)= & \frac{1}{2}\left[\operatorname{th} \frac{\beta}{2}\left(\frac{J_{2}}{2} \xi_{2 p}(2)-2 \psi_{5} \varepsilon_{5}+\mu_{2} E_{2}+\frac{J_{4}}{2} \sigma_{p}(2)+\Delta\right)\right. \\
& \left.-\operatorname{th} \frac{\beta}{2}\left(\frac{J_{2}}{2} \xi_{2 p}(2)-2 \psi_{5} \varepsilon_{5}+\mu_{2} E_{2}-\frac{J_{4}}{2} \sigma_{p}(2)-\Delta\right)\right] .
\end{aligned}
$$

If $E_{3} \neq 0$ and $\sigma_{6} \neq 0 \eta_{1}=-\eta_{4}=\eta_{14}$ and $\eta_{2}=-\eta_{3}=\eta_{23}$. As a result

$$
\begin{aligned}
\xi_{3 p}(3)= & \frac{1}{2}\left[\operatorname{th} \frac{\beta}{2}\left(\frac{J_{3}}{2} \xi_{3 p}(3)-2 \psi_{6} \varepsilon_{6}+\mu_{3} E_{3} \frac{J_{4}}{2} \sigma_{p}(3)+\Delta\right)\right. \\
& \left.+\operatorname{th} \frac{\beta}{2}\left(\frac{J_{3}}{2} \xi_{3 p}(3)-2 \psi_{6} \varepsilon_{6}+\mu_{3} E_{3}-\frac{J_{4}}{2} \sigma_{p}(3)-\Delta\right)\right], \\
\sigma_{p}(3)= & \frac{1}{2}\left[\operatorname{th} \frac{\beta}{2}\left(\frac{J_{3}}{2} \xi_{3 p}(3)-2 \psi_{6} \varepsilon_{6}+\mu_{3} E_{3}+\frac{J_{4}}{2} \sigma_{p}(3)+\Delta\right)\right. \\
& \left.-\operatorname{th} \frac{\beta}{2}\left(\frac{J_{3}}{2} \xi_{3 p}(3)-2 \psi_{6} \varepsilon_{6}+\mu_{3} E_{3}-\frac{J_{4}}{2} \sigma_{p}(3)-\Delta\right)\right] .
\end{aligned}
$$

To go to the Mitsui model we need to go from the four-sublattice model to the two-sublattice one $\left(v_{M}=v / 2\right)$ and at $E_{2}=E_{3}=0$ in all phases $\xi_{2}(0)=0$ and $\xi_{3}(0)=0$. Also $J_{M}=J+K_{12}$ and $K_{M}=K_{13}+K_{14}$.

\section{Thermodynamic characteristics of Rochelle salt}

To obtain expressions for dielectric, piezoelectric, elastic characteristics of Rochelle salt we use a thermodynamic potential per one unit calculated cell in the mean field approximation

$$
\begin{aligned}
g= & \frac{G}{N k_{\mathrm{B}}}=\frac{\bar{v}}{2} c_{44}^{E 0} \varepsilon_{4}^{2}+\frac{\bar{v}}{2} c_{55}^{E 0} \varepsilon_{5}^{2}+\frac{\bar{v}}{2} c_{66}^{E 0} \varepsilon_{6}^{2}-\bar{v} e_{14}^{0} \varepsilon_{4} E_{1}-\bar{v} e_{25}^{0} \varepsilon_{5} E_{2}-\bar{v} e_{36}^{0} \varepsilon_{6} E_{3} \\
& -\frac{\bar{v}}{2} \chi_{11}^{\varepsilon 0} E_{1}^{2}-\frac{\bar{v}}{2} \chi_{22}^{\varepsilon 0} E_{2}^{2}-\frac{\bar{v}}{2} \chi_{33}^{\varepsilon 0} E_{3}^{2}-4 T \ln 2+\frac{\tilde{J}_{1}}{2} \xi_{1}^{2}+\frac{\tilde{J}_{2}}{2} \xi_{2}^{2}+\frac{\tilde{J}_{3}}{2} \xi_{3}^{2}+\frac{\tilde{J}_{4}}{2} \sigma^{2} \\
& -T \sum_{f=1}^{4} \ln \operatorname{ch} \frac{\beta}{2} \mathcal{H}_{f}-\bar{v} \sum_{j=4}^{6} \sigma_{j} \varepsilon_{j},
\end{aligned}
$$


where $\tilde{J}_{i}=\frac{J_{i}}{k_{\mathrm{B}}}$.

From the conditions of equilibrium

$$
\frac{1}{\bar{v}}\left(\frac{\partial g}{\partial \varepsilon_{j}}\right)_{E_{i}, \sigma_{j}}=0, \quad \frac{1}{\bar{v}}\left(\frac{\partial g}{\partial E_{i}}\right)=-P_{i}
$$

we obtain

$$
\begin{gathered}
\sigma_{j}=c_{j j}^{E 0} \varepsilon_{j}-e_{i j}^{0} E_{i}+4 \frac{\psi_{j}}{v} \xi_{i}, \\
P_{i}=e_{i j}^{0} \varepsilon_{j}+\chi_{i i}^{\varepsilon 0} E_{i}+2 \frac{\mu_{i}}{v} \xi_{i} .
\end{gathered}
$$

From (3.3) the electric fields are

$$
E_{i}=-h_{i j}^{0} \varepsilon_{j}+k_{i i}^{\varepsilon 0}\left(P_{i}-2 \frac{\mu_{i}}{v} \xi_{i}\right),
$$

where $h_{i j}^{0}=\frac{e_{i j}^{0}}{\chi_{i i}^{\varepsilon 0}}, k_{i i}^{\varepsilon 0}=\frac{1}{\chi_{i i}^{\varepsilon 0}}$.

Substituting (3.4) into (3.2), we get

$$
\sigma_{j}=c_{j j}^{P 0} \varepsilon_{j}-h_{i j}^{0}\left(P_{i}-2 \frac{\mu_{i}}{v} \xi_{i}\right)+\frac{4 \psi_{i}}{v} \xi_{i},
$$

where $c_{j j}^{P 0}=c_{j j}^{E 0}+e_{i j}^{0} h_{i j}^{0}$.

Using expressions (2.8) and (3.3), we calculate static dielectric susceptibilities of mechanically clamped crystal of Rochelle salt

$$
\chi_{i i}^{\varepsilon}=\left(\frac{\partial P_{i}}{\partial E_{i}}\right)_{\varepsilon_{j}}=\chi_{i i}^{\varepsilon 0}+2 \frac{\mu_{i}}{v}\left(\frac{\partial \xi_{i}}{\partial E_{i}}\right)_{\varepsilon_{j}} .
$$

The static dielectric susceptibilities along the $a$ and $b$ axes, dependent on the stress $\sigma_{5}$ and field $E_{2}$, in the paraelectric phase read

$$
\begin{aligned}
& \chi_{11 p}^{\varepsilon}\left(\sigma_{5}, E_{2}\right)=\chi_{11}^{\varepsilon 0}+\frac{\mu_{1}^{2}}{v} \beta F_{11 p}\left(\xi_{2 p}(2) \sigma_{p}(2)\right), \\
& \chi_{22 p}^{\varepsilon}\left(\sigma_{5}, E_{2}\right)=\chi_{22}^{\varepsilon 0}+\frac{\mu_{2}^{2}}{v} \beta F_{12 p}\left(\xi_{2 p}(2) \sigma_{p}(2)\right),
\end{aligned}
$$

where the notations are used

$$
\begin{aligned}
& F_{11 p}\left(\xi_{2 p}(2) \sigma_{p}(2)\right)=\frac{\rho_{1 p}(2)-\left(\rho_{1 p}^{2}(2)-\rho_{3 p}^{2}(2)\right) \frac{\beta J_{3}}{4}}{1-\rho_{1 p}(2)\left(\frac{\beta J_{1}}{4}+\frac{\beta J_{3}}{4}\right)+\left(\rho_{1 p}^{2}(2)-\rho_{3 p}^{2}(2)\right) \frac{\beta J_{1}}{4} \frac{\beta J_{3}}{4}}, \\
& F_{12 p}\left(\xi_{2 p}(2) \sigma_{p}(2)\right)=\frac{\rho_{1 p}(2)-\left(\rho_{1 p}^{2}(2)-\rho_{3 p}^{2}(2)\right) \frac{\beta J_{4}}{4}}{1-\rho_{1 p}(2)\left(\frac{\beta J_{2}}{4}+\frac{\beta J_{4}}{4}\right)+\left(\rho_{1 p}^{2}(2)-\rho_{3 p}^{2}(2)\right) \frac{\beta J_{2}}{4} \frac{\beta J_{4}}{4}}, \\
& \rho_{1 p}(2)=1-\xi_{2 p}^{2}(2)-\sigma_{p}^{2}(2), \quad \rho_{3 p}(2)=2 \xi_{2 p}(2) \sigma_{p}(2) .
\end{aligned}
$$

The static dielectric susceptibilities along the $a$ and $b$ axes, dependent on the stress $\sigma_{6}$ and field $E_{3}$, in the paraelectric phase read

$$
\begin{aligned}
& \chi_{11 p}^{\varepsilon}\left(\sigma_{6}, E_{3}\right)=\chi_{11}^{\varepsilon 0}+\frac{\mu_{1}^{2}}{v} \beta F_{11 p}\left(\xi_{3 p}(3) \sigma_{p}(3)\right), \\
& \chi_{33 p}^{\varepsilon}\left(\sigma_{6}, E_{3}\right)=\chi_{33}^{\varepsilon 0}+\frac{\mu_{3}^{2}}{v} \beta F_{13 p}\left(\xi_{3 p}(3) \sigma_{p}(3)\right),
\end{aligned}
$$


where

$$
\begin{aligned}
& F_{11 p}\left(\xi_{3 p}(3) \sigma_{p}(3)\right)=\frac{\rho_{1 p}(3)-\left(\rho_{1 p}^{2}(3)-\rho_{2 p}^{2}(3)\right) \frac{\beta J_{2}}{4}}{1-\rho_{1 p}(3)\left(\frac{\beta J_{1}}{4}+\frac{\beta J_{2}}{4}\right)+\left(\rho_{1 p}^{2}(3)-\rho_{2 p}^{2}(3)\right) \frac{\beta J_{1}}{4} \frac{\beta J_{2}}{4}}, \\
& F_{13 p}\left(\xi_{3 p}(3) \sigma_{p}(3)\right)=\frac{\rho_{1 p}(3)-\left(\rho_{1 p}^{2}(3)-\rho_{2 p}^{2}(3)\right) \frac{\beta J_{4}}{4}}{1-\rho_{1 p}(3)\left(\frac{\beta J_{3}}{4}+\frac{\beta J_{4}}{4}\right)+\left(\rho_{1 p}^{2}(3)-\rho_{2 p}^{2}(3)\right) \frac{\beta J_{3}}{4} \frac{\beta J_{4}}{4}},
\end{aligned}
$$

and $\rho_{1 p}(3)=1-\xi_{3 p}^{2}(3)-\sigma_{p}^{2}(3), \rho_{2 p}(3)=2 \xi_{3 p}(3) \sigma_{p}(3)$.

In the ferroelectric phase

$$
\begin{aligned}
& \chi_{11 s}^{\varepsilon}\left(\sigma_{5}, E_{2}\right)=\chi_{11}^{\varepsilon 0}+\frac{\mu_{1}^{2}}{v} \beta F_{11 s}\left(\xi_{1 s}(2), \xi_{2 s}(2), \xi_{3 s}(2), \sigma_{s}(2)\right), \\
& \chi_{22 s}^{\varepsilon}\left(\sigma_{5}, E_{2}\right)=\chi_{11}^{\varepsilon 0}+\frac{\mu_{2}^{2}}{v} \beta F_{12 s}\left(\xi_{1 s}(2), \xi_{2 s}(2), \xi_{3 s}(2), \sigma_{s}(2)\right),
\end{aligned}
$$

where

$$
\begin{aligned}
& F_{11 s}\left(\xi_{1 s}(2), \xi_{2 s}(2), \xi_{3 s}(2), \sigma_{s}(2)\right)=\frac{\Delta_{1}^{\varepsilon}(2)}{\Delta^{\varepsilon}(2)}, \\
& F_{12 s}\left(\xi_{1 s}(2), \xi_{2 s}(2), \xi_{3 s}(2), \sigma_{s}(2)\right)=\frac{\Delta_{2}^{\varepsilon}(2)}{\Delta^{\varepsilon}(2)},
\end{aligned}
$$

and

$$
\begin{aligned}
& \Delta^{\varepsilon}(2)=\left|\begin{array}{cccc}
1-\rho_{1 s}(2) \frac{\beta J_{1}}{4} & \rho_{2 s}(2) \frac{\beta J_{2}}{4} & \rho_{3 s}(2) \frac{\beta J_{3}}{4} & \rho_{4 s}(2) \frac{\beta J_{4}}{4} \\
\rho_{2 s}(2) \frac{\beta J_{1}}{4} & 1-\rho_{1 s}(2) \frac{\beta J_{2}}{4} & \rho_{4 s}(2) \frac{\beta J_{3}}{4} & \rho_{3 s}(2) \frac{\beta J_{4}}{4} \\
\rho_{3 s}(2) \frac{\beta J_{1}}{4} & \rho_{4 s}(2) \frac{\beta J_{2}}{4} & 1-\rho_{1 s}(2) \frac{\beta J_{3}}{4} & \rho_{2 s}(2) \frac{\beta J_{4}}{4} \\
\rho_{4 s}(2) \frac{\beta J_{1}}{4} & \rho_{3 s}(2) \frac{\beta J_{2}}{4} & \rho_{2 s}(2) \frac{\beta J_{3}}{4} & 1-\rho_{1 s}(2) \frac{\beta J_{4}}{4}
\end{array}\right|, \\
& \Delta_{1}^{\varepsilon}(2)=\left|\begin{array}{cccc}
\rho_{1 s}(2) & \rho_{2 s}(2) \frac{\beta J_{2}}{4} & \rho_{3 s}(2) \frac{\beta J_{3}}{4} & \rho_{4 s}(2) \frac{\beta J_{4}}{4} \\
-\rho_{2 s}(2) & 1-\rho_{1 s}(2) \frac{\beta J_{2}}{4} & \rho_{4 s}(2) \frac{\beta J_{4}}{4} & \rho_{3 s}(2) \frac{\beta J_{4}}{4} \\
-\rho_{3 s}(2) & \rho_{4 s}(2) \frac{\beta J_{2}}{4} & 1-\rho_{1 s}(2) \frac{\beta J_{3}}{4} & \rho_{2 s}(2) \frac{\beta J_{4}}{4} \\
-\rho_{4 s}(2) & \rho_{3 s}(2) \frac{\beta J_{2}}{4} & \rho_{2 s}(2) \frac{\beta J_{3}}{4} & 1-\rho_{1 s}(2) \frac{\beta J_{4}}{4}
\end{array}\right|, \\
& \Delta_{2}^{\varepsilon}(2)=\left|\begin{array}{cccc}
1-\rho_{1 s}(2) \frac{\beta J_{1}}{4} & -\rho_{2 s}(2) & \rho_{3 s}(2) \frac{\beta J_{3}}{4} & \rho_{4 s}(2) \frac{\beta J_{4}}{4} \\
\rho_{2 s}(2) \frac{\beta J_{1}}{4} & \rho_{1 s}(2) & \rho_{4 s}(2) \frac{\beta J_{3}}{4} & \rho_{3 s}(2) \frac{\beta J_{4}}{4} \\
\rho_{3 s}(2) \frac{\beta J_{1}}{4} & -\rho_{4 s}(2) & 1-\rho_{1 s}(2) \frac{\beta J_{3}}{4} & \rho_{2 s}(2) \frac{\beta J_{4}}{4} \\
\rho_{4 s}(2) \frac{\beta J_{1}}{4} & -\rho_{3 s}(2) & \rho_{2 s}(2) & 1-\rho_{1 s}(2) \frac{\beta J_{4}}{4}
\end{array}\right|,
\end{aligned}
$$

where the following notations are used

$$
\begin{aligned}
& \rho_{1 s}(2)=1-\xi_{1 s}^{2}(2)-\xi_{2 s}^{2}(2)-\xi_{3 s}^{2}(2)-\sigma_{s}^{2}(2), \\
& \rho_{2 s}(2)=2 \xi_{1 s}(2) \xi_{2 s}(2)+2 \xi_{3 s}(2) \sigma_{s}(2), \\
& \rho_{3 s}(2)=2 \xi_{1 s}(2) \xi_{3 s}(2)+2 \xi_{2 s}(2) \sigma_{s}(2), \\
& \rho_{4 s}(2)=2 \xi_{2 s}(2) \xi_{3 s}(2)+2 \xi_{1 s}(2) \sigma_{s}(2),
\end{aligned}
$$

and $\xi_{1 s}(2), \ldots, \sigma_{s}(2)$ are obtained from $(2.8)$ at

$$
\begin{array}{ll}
\gamma_{1}(2)=\beta\left(\frac{J_{1}}{2} \xi_{1 s}(2)-2 \psi_{4} \varepsilon_{4}\right), & \gamma_{2}(2)=\beta\left(\frac{J_{2}}{2} \xi_{2 s}(2)-2 \psi_{5} \varepsilon_{5}+\mu_{2} E_{2}\right), \\
\gamma_{3}(2)=\frac{\beta J_{3}}{2} \xi_{3 s}(2), & \delta(2)=\beta\left(\frac{J_{4}}{2} \sigma_{s}(2)+\Delta\right) .
\end{array}
$$


Similarly

$$
\begin{aligned}
& \chi_{11 s}^{\varepsilon}\left(\sigma_{6}, E_{3}\right)=\chi_{11}^{\varepsilon 0}+\frac{\mu_{1}^{2}}{v} \beta F_{11 s}\left(\xi_{1 s}(3), \xi_{2 s}(3), \xi_{3 s}(3), \sigma_{s}(3)\right), \\
& \chi_{33 s}^{\varepsilon}\left(\sigma_{6}, E_{3}\right)=\chi_{33}^{\varepsilon 0}+\frac{\mu_{3}^{2}}{v} \beta F_{13 s}\left(\xi_{1 s}(3), \xi_{2 s}(3), \xi_{3 s}(3), \sigma_{s}(3)\right),
\end{aligned}
$$

where

$$
\begin{aligned}
& F_{11 s}\left(\xi_{1 s}(3), \xi_{2 s}(3), \xi_{3 s}(3), \sigma_{s}(3)\right)=\frac{\Delta_{1}^{\varepsilon}(3)}{\Delta^{\varepsilon}(3)} \\
& F_{13 s}\left(\xi_{1 s}(3), \xi_{2 s}(3), \xi_{3 s}(3), \sigma_{s}(3)\right)=\frac{\Delta_{3}^{\varepsilon}(3)}{\Delta^{\varepsilon}(3)}
\end{aligned}
$$

and

$$
\Delta_{3}^{\varepsilon}(3)=\left|\begin{array}{cccc}
1-\rho_{1 s}(3) \frac{\beta J_{1}}{4} & \rho_{2 s}(3) \frac{\beta J_{2}}{4} & -\rho_{3 s}(3) & \rho_{4 s}(3) \frac{\beta J_{4}}{4} \\
\rho_{2 s}(3) \frac{\beta J_{1}}{4} & 1-\rho_{1 s}(3) \frac{\beta J_{2}}{4} & -\rho_{4 s}(3) & \rho_{3 s}(3) \frac{\beta J_{4}}{4} \\
\rho_{3 s}(3) \frac{\beta J_{1}}{4} & \rho_{4 s}(3) \frac{\beta J_{2}}{4} & \rho_{1 s}(3) & \rho_{2 s}(3) \frac{\beta J_{4}}{4} \\
\rho_{4 s}(3) \frac{\beta J_{1}}{4} & \rho_{3 s}(3) \frac{\beta J_{2}}{4} & -\rho_{2 s}(3) & 1-\rho_{1 s}(3) \frac{\beta J_{4}}{4}
\end{array}\right| .
$$

Expressions for $\xi_{1 s}(3), \ldots, \sigma_{s}(3)$, entering $\rho_{f s}(3)$, are obtained from (2.8) at

$$
\begin{aligned}
& \gamma_{1}(3)=\beta\left(\frac{J_{1}}{2} \xi_{1 s}(3)-2 \psi_{4} \varepsilon_{4}\right), \quad \gamma_{2}(3)=\beta \frac{J_{2}}{2} \xi_{2 s}(3), \\
& \gamma_{3}(3)=\beta\left(\frac{J_{3}}{2} \xi_{3 s}(3)-2 \psi_{6} \varepsilon_{6}+\mu_{3} E_{3}\right), \quad \delta(3)=\beta\left(\frac{J_{4}}{2} \sigma_{s}(3)+\Delta\right) .
\end{aligned}
$$

From relations (2.8) and (3.3) we derive expressions for the coefficients of piezoelectric stress $e_{i j}$ of deuterated Rochelle salt

$$
\begin{array}{rlrl}
e_{i j p}\left(\sigma_{5}, E_{2}\right)=\left(\frac{\partial P_{i}}{\partial \varepsilon_{j}}\right)_{E_{i}}=e_{i j}^{0}-\frac{\mu_{i}}{v} 2 \psi_{j} \beta F_{1 i p}\left(\xi_{2 p}(2), \sigma_{p}(2)\right), & (i=1,2), \\
e_{i j p}\left(\sigma_{6}, E_{3}\right)=\left(\frac{\partial P_{i}}{\partial \varepsilon_{j}}\right)_{E_{i}}=e_{i j}^{0}-\frac{\mu_{i}}{v} 2 \psi_{j} \beta F_{1 i p}\left(\xi_{3 p}(3), \sigma_{p}(3)\right), & (i=1,3), \\
e_{i j s}\left(\sigma_{5}, E_{2}\right)=e_{i j}^{0}-\frac{\mu_{i}}{v} 2 \psi_{j} \beta F_{1 i s}\left(\xi_{1 s}(2), \xi_{2 s}(2), \xi_{3 s}(2), \sigma_{s}(2)\right), & & (i=1,2), \\
e_{i j s}\left(\sigma_{6}, E_{3}\right)=e_{i j}^{0}-\frac{\mu_{i}}{v} 2 \psi_{j} \beta F_{1 i s}\left(\xi_{1 s}(3), \xi_{2 s}(3), \xi_{3 s}(3), \sigma_{s}(3)\right), & & (i=1,3) .
\end{array}
$$

Differentiating (3.4) with respect to the strain at constant polarization we obtain expressions for the constants of piezoelectric stress

$$
h_{i j}=-\left(\frac{\partial E_{i}}{\partial \varepsilon_{j}}\right)_{P_{i}}=\frac{e_{i j}}{\chi_{11}^{\varepsilon}} .
$$

Let us now calculate the contributions of the pseudospin subsystem to the elastic constants. From (2.8) and (3.2) we obtain expressions for the elastic constants at a constant field

$$
\begin{array}{rlrl}
c_{j j p}^{E}\left(\sigma_{5}, E_{2}\right)=\left(\frac{\partial \sigma_{j}}{\partial \varepsilon_{j}}\right)_{E_{i}}=c_{j j}^{E 0}-\frac{4 \psi_{j}^{2}}{v} \beta F_{1 i p}\left(\xi_{2 p}(2), \sigma_{p}(2)\right), & & (i=1,2), \\
c_{j j p}^{E}\left(\sigma_{6}, E_{3}\right)=c_{j j}^{E 0}-\frac{4 \psi_{j}^{2}}{v} \beta F_{1 i p}\left(\xi_{3 p}(3), \sigma_{p}(3)\right), & (i=1,3), \\
c_{j j s}^{E}\left(\sigma_{5}, E_{2}\right)=c_{j j}^{E 0}-\frac{4 \psi_{j}^{2}}{v} \beta F_{1 i s}\left(\xi_{1 s}(2), \xi_{2 s}(2), \xi_{3 s}(2), \sigma_{s}(2)\right), & (i=1,2), \\
c_{j j s}^{E}\left(\sigma_{6}, E_{3}\right)=c_{j j}^{E 0}-\frac{4 \psi_{j}^{2}}{v} \beta F_{1 i s}\left(\xi_{1 s}(3), \xi_{2 s}(3), \xi_{3 s}(3), \sigma_{s}(3)\right), & (i=1,3),
\end{array}
$$


and at a constant polarization

$$
c_{j j}^{P}=c_{j j}^{E}+e_{i j} h_{i j}, \quad(i=1,2,3) .
$$

Let us find the strains $\varepsilon_{j}$ from $(3.2)$

$$
\varepsilon_{j}=s_{j j}^{E 0} \sigma_{j}+d_{i j}^{0} E_{i}-4 \frac{\psi_{j}}{v} s_{j j}^{E 0} \xi_{i},
$$

where $s_{j j}^{E 0}=\left(c_{j j}^{E 0}\right)^{-1}, d_{i j}^{0}=e_{i j}^{0} s_{j j}^{E 0}$.

Substituting expressions (3.13) to (3.1), we find the Gibbs' function

$$
\begin{aligned}
g= & -\frac{\bar{v}}{2} s_{44}^{E 0} \sigma_{4}^{2}-\frac{\bar{v}}{2} s_{55}^{E 0} \sigma_{5}^{2}-\frac{\bar{v}}{2} s_{66}^{E 0} \sigma_{6}^{2}-\bar{v} d_{14}^{0} \sigma_{4} E_{1}-\bar{v} d_{25}^{0} \sigma_{5} E_{2}-\bar{v} d_{36}^{0} \sigma_{6} E_{3}-\frac{\bar{v}}{2} \chi_{11}^{\sigma 0} E_{1}^{2}-\frac{\bar{v}}{2} \chi_{22}^{\sigma 0} E_{2}^{2} \\
& -\frac{\bar{v}}{2} \chi_{33}^{\sigma 0} E_{3}^{2}-4 T \ln 2+\tilde{J}_{1} \bar{\xi}_{1}^{2}+\tilde{J}_{2} \bar{\xi}_{2}^{2}+\tilde{J}_{3} \bar{\xi}_{3}^{2}+\tilde{J}_{4} \bar{\sigma}^{2}-T \sum_{f=1}^{4} \ln \operatorname{ch} \frac{\beta}{2} \overline{\mathcal{H}}_{f}
\end{aligned}
$$

where

$$
\begin{gathered}
\chi_{i i}^{\sigma 0}=\chi_{i i}^{\varepsilon 0}+e_{i j}^{0} d_{i j}^{0}, \\
\overline{\mathcal{H}}_{1}=\frac{1}{\beta}\left(\bar{\gamma}_{1} \pm \bar{\gamma}_{2}+\bar{\gamma}_{3} \pm \bar{\delta}\right), \quad \overline{\mathcal{H}}_{2}=\frac{1}{\beta}\left(\bar{\gamma}_{1} \mp \bar{\gamma}_{2}-\bar{\gamma}_{3} \pm \bar{\delta}\right) \\
\bar{\gamma}_{i}=\beta\left[\left(\frac{J_{i}}{2}+8 \frac{\psi_{j}^{2}}{v} s_{j j}^{E 0}\right) \bar{\xi}_{i}-2 \psi_{j} s_{j j}^{E 0} \sigma_{j}+\left(\mu_{i}-2 \psi_{j} d_{j j}^{0}\right)\right), \quad \bar{\delta}=\beta\left(\frac{J_{4}}{2} \bar{\sigma}+\Delta\right) .
\end{gathered}
$$

From conditions of thermodynamic equilibrium

$$
\frac{1}{\bar{v}}\left(\frac{\partial g_{1 E}}{\partial \sigma_{j}}\right)_{E_{i}}=-\varepsilon_{j}, \quad \frac{1}{\bar{v}}\left(\frac{\partial g_{1 E}}{\partial E_{j}}\right)=-P_{i}
$$

we find relations (3.13) and

$$
P_{i}=d_{i j}^{0} \sigma_{j}+\chi_{i i}^{\sigma 0} E_{i}+2\left(\frac{\mu_{i}}{v}-2 \frac{\psi_{j}}{v} d_{i j}^{0}\right) \bar{\xi}_{i} .
$$

Thence

$$
E_{i}=-g_{i j}^{0} \sigma_{j}+k_{i i}^{\sigma 0} P_{i}+\left(4 \frac{\psi_{j}}{v} g_{i j}^{0}-2 k_{i i}^{\sigma 0} \frac{\mu_{i}}{v}\right) \bar{\xi}_{i},
$$

where $k_{i i}^{\sigma 0}=\left(\chi_{i i}^{\sigma 0}\right)^{-1}, g_{i j}^{0}=d_{i j}^{0} k_{i i}^{\sigma 0}$.

Substituting (3.16) into (3.13), we get

$$
\varepsilon_{j}=s_{j j}^{P 0} \sigma_{j}+g_{i j}^{0} P_{i}-\left(\frac{\psi_{j}}{v} s_{j j}^{P 0}+g_{i j}^{0} 2 \frac{\mu_{i}}{v}\right) \bar{\xi}_{i}
$$

where

$$
s_{j j}^{P 0}=s_{j j}^{E 0}-g_{i j}^{0} d_{i j}^{0} .
$$

Using expressions (3.15), we find static dielectric susceptibilities of mechanically free crystal of Rochelle salt

$$
\begin{aligned}
\chi_{i i p}^{\sigma}\left(\sigma_{5}, E_{2}\right) & =\chi_{i i}^{\sigma 0}+\frac{\bar{\mu}_{i}^{2}}{v} \beta F_{2 i p}\left(\bar{\xi}_{2 p}(2), \bar{\sigma}_{p}(2)\right), \quad(i=1,2), \\
\chi_{i i p}^{\sigma}\left(\sigma_{6}, E_{3}\right) & =\chi_{i i}^{\sigma 0}+\frac{\bar{\mu}_{i}^{2}}{v} \beta F_{2 i p}\left(\bar{\xi}_{3 p}(3), \bar{\sigma}_{p}(3)\right), \quad(i=1,3), \\
\chi_{i i s}^{\sigma}\left(\sigma_{5}, E_{2}\right) & =\chi_{i i}^{\sigma 0}+\frac{\bar{\mu}_{i}^{2}}{v} \beta F_{2 i s}\left(\bar{\xi}_{1 s}(2), \bar{\xi}_{2 s}(2), \bar{\xi}_{3 s}(2), \bar{\sigma}_{s}(2)\right), \quad(i=1,2), \\
\chi_{i i s}^{\sigma}\left(\sigma_{6}, E_{3}\right) & =\chi_{i i}^{\sigma 0}+\frac{\bar{\mu}_{i}^{2}}{v} \beta F_{2 i s}\left(\bar{\xi}_{1 s}(3), \bar{\xi}_{2 s}(3), \bar{\xi}_{3 s}(3), \bar{\sigma}_{s}(3)\right), \quad(i=1,3),
\end{aligned}
$$


where we use the following notations

$$
\begin{aligned}
& \bar{\mu}_{i}=\mu_{i}-2 \psi_{j} d_{i j}^{0}, \\
& F_{21 p}\left(\bar{\xi}_{2 p}(2), \bar{\sigma}_{p}(2)\right)=\frac{\bar{\rho}_{1 p}(2)-\left(\bar{\rho}_{1 p}^{2}(2)-\bar{\rho}_{3 p}^{2}(2)\right) \frac{\beta J_{3}}{4}}{1-\bar{\rho}_{1 p}(2)\left(\frac{\beta J_{1}}{4}+4 \frac{\psi_{4}^{2}}{v T} s_{44}^{E 0}+\frac{\beta J_{3}}{4}\right)+\left(\bar{\rho}_{1 p}^{2}(2)-\bar{\rho}_{3 p}^{2}(2)\right)\left(\frac{\beta J_{1}}{4}+4 \frac{\psi_{4}^{2}}{v T} s_{44}^{E 0}\right) \frac{\beta J_{3}}{4}}, \\
& F_{22 p}\left(\bar{\xi}_{2 p}(2), \bar{\sigma}_{p}(2)\right)=\frac{\bar{\rho}_{1 p}(2)-\left(\bar{\rho}_{1 p}^{2}(2)-\bar{\rho}_{3 p}^{2}(2)\right) \frac{\beta J_{4}}{4}}{1-\bar{\rho}_{1 p}(2)\left(\frac{\beta J_{2}}{4}+4 \frac{\psi_{5}^{2}}{v T} s_{55}^{E 0}+\frac{\beta J_{4}}{4}\right)+\left(\bar{\rho}_{1 p}^{2}(2)-\bar{\rho}_{3 p}^{2}(2)\right)\left(\frac{\beta J_{2}}{4}+4 \frac{\psi_{5}^{2}}{v T} s_{55}^{E 0}\right) \frac{\beta J_{4}}{4}}, \\
& \bar{\rho}_{1 p}(2)=1-\bar{\xi}_{2 p}(2)-\bar{\sigma}_{p}^{2}(2), \quad \bar{\rho}_{3 p}(2)=2 \bar{\xi}_{2 p}(2) \bar{\sigma}_{p}(2) \\
& \bar{\xi}_{2 p}(2)=\frac{1}{2}\left[\operatorname{th} \frac{\beta}{2}\left(\bar{\gamma}_{2}+\bar{\delta}\right)+\operatorname{th} \frac{\beta}{2}\left(\bar{\gamma}_{2}-\bar{\delta}\right)\right] \\
& \bar{\sigma}_{p}(2)=\frac{1}{2}\left[\operatorname{th} \frac{\beta}{2}\left(\bar{\gamma}_{2}+\bar{\delta}\right)-\operatorname{th} \frac{\beta}{2}\left(\bar{\gamma}_{2}-\bar{\delta}\right)\right] ; \\
& F_{21 p}\left(\bar{\xi}_{2 p}(3), \bar{\sigma}_{p}(3)\right)=\frac{\bar{\rho}_{1 p}(3)-\left(\bar{\rho}_{1 p}^{2}(3)-\bar{\rho}_{2 p}^{2}(3)\right) \frac{\beta J_{2}}{4}}{1-\bar{\rho}_{1 p}(3)\left(\frac{\beta J_{1}}{4}+4 \frac{\psi_{4}^{2}}{v T} s_{44}^{E 0}+\frac{\beta J_{2}}{4}\right)+\left(\bar{\rho}_{1 p}^{2}(3)-\bar{\rho}_{2 p}^{2}(3)\right)\left(\frac{\beta J_{1}}{4}+4 \frac{\psi_{4}^{2}}{v T} s_{44}^{E 0}\right) \frac{\beta J_{2}}{4}} \\
& F_{23 p}\left(\bar{\xi}_{2 p}(3), \bar{\sigma}_{p}(3)\right)=\frac{\bar{\rho}_{1 p}(3)-\left(\bar{\rho}_{1 p}^{2}(3)-\bar{\rho}_{2 p}^{2}(3)\right) \frac{\beta J_{4}}{4}}{1-\bar{\rho}_{1 p}(3)\left(\frac{\beta J_{3}}{4}+4 \frac{\psi_{6}^{2}}{v T} s_{66}^{E 0}+\frac{\beta J_{4}}{4}\right)+\left(\bar{\rho}_{1 p}^{2}(3)-\bar{\rho}_{2 p}^{2}(3)\right)\left(\frac{\beta J_{3}}{4}+4 \frac{\psi_{6}^{2}}{v T} s_{66}^{E 0}\right) \frac{\beta J_{4}}{4}},
\end{aligned}
$$

where

$$
\begin{aligned}
& \bar{\rho}_{1 p}(3)=1-\bar{\xi}_{3 p}(3)-\bar{\sigma}_{p}^{2}(3), \quad \bar{\rho}_{2 p}(3)=2 \bar{\xi}_{3 p}(3) \bar{\sigma}_{p}(3), \\
& \bar{\xi}_{3 p}(3)=\frac{1}{2}\left[\operatorname{th} \frac{\beta}{2}\left(\bar{\gamma}_{3}+\bar{\delta}\right)+\operatorname{th} \frac{\beta}{2}\left(\bar{\gamma}_{3}-\bar{\delta}\right)\right], \\
& \bar{\sigma}_{p}(3)=\frac{1}{2}\left[\operatorname{th} \frac{\beta}{2}\left(\bar{\gamma}_{3}+\bar{\delta}\right)-\operatorname{th} \frac{\beta}{2}\left(\bar{\gamma}_{3}-\bar{\delta}\right)\right] ; \\
& F_{2 i s}\left(\bar{\xi}_{1 s}(2), \bar{\xi}_{2 s}(2), \bar{\xi}_{3 s}(2), \bar{\sigma}_{s}(2)\right)=\frac{\Delta_{i}^{\sigma}(2)}{\Delta^{\sigma}(2)}, \quad(i=1,2),
\end{aligned}
$$

the determinants $\Delta^{\sigma}(2), \Delta_{i}^{\sigma}(2)$ are obtained from $\Delta^{\varepsilon}(2), \Delta_{i}^{\varepsilon}(2)$ by replacing $\rho_{f s}(2)$ with $\bar{\rho}_{f s}(2)$, $J_{1}$ and $J_{2}$ with $\bar{J}_{1}$ and $\bar{J}_{2}$, respectively, where

$$
\bar{J}_{1}=J_{1}+16 \frac{\psi_{4} \psi_{4}}{v} s_{44}^{E 0}, \quad \bar{J}_{2}=J_{2}+16 \frac{\psi_{5} \psi_{5}}{v} s_{55}^{E 0},
$$

and $\bar{\xi}_{1 s}(2), \ldots, \bar{\sigma}_{s}(2)$ are obtained from $(2.8)$ at

$$
\begin{aligned}
& \bar{\gamma}_{1}(2)=\beta\left(\frac{J_{1}}{2}+8 \frac{\psi_{4}^{2}}{v} s_{44}^{E 0}\right) \bar{\xi}_{1 s}(2), \quad \bar{\gamma}_{2}(2)=\beta\left[\left(\frac{J_{2}}{2}+8 \frac{\psi_{5}^{2}}{v} s_{55}^{E 0}\right) \bar{\xi}_{2 s}(2)-2 \psi_{5} s_{55}^{E 0} \sigma_{5}+\bar{\mu}_{2} E_{2}\right], \\
& \bar{\gamma}_{3}(2)=\beta \frac{J_{3}}{2} \bar{\xi}_{3 s}(2), \quad \bar{\delta}(2)=\beta\left(\frac{J_{4}}{2} \bar{\sigma}_{s}(2)+\Delta\right) ; \\
& F_{2 i s}\left(\bar{\xi}_{1 s}(3), \bar{\xi}_{2 s}(3), \bar{\xi}_{3 s}(3), \bar{\sigma}_{s}(3)\right)=\frac{\Delta_{i}^{\sigma}(3)}{\Delta^{\sigma}(3)}, \quad(i=1,3),
\end{aligned}
$$

the determinants $\Delta^{\sigma}(3), \Delta_{i}^{\sigma}(3)$ are obtained from $\Delta^{\varepsilon}(2)$ and $\Delta_{i}^{\varepsilon}(2)$ by replacing $\rho_{f s}(2)$ with $\bar{\rho}_{f s}(3)$, $J_{1}$ and $J_{3}$ with $\bar{J}_{1}$ and $\bar{J}_{3}$, where

$$
\bar{J}_{3}=J_{3}+16 \frac{\psi_{6} \psi_{6}}{v} s_{66}^{E 0},
$$


whereas $\bar{\xi}_{1 s}(3), \ldots, \bar{\sigma}_{s}(3)$ are determined from $(2.8)$ at

$$
\begin{aligned}
& \bar{\gamma}_{1}(3)=\beta\left(\frac{J_{1}}{2}+8 \frac{\psi_{4}^{2}}{v} s_{44}^{E 0}\right) \bar{\xi}_{1 s}(3), \quad \bar{\gamma}_{2}(3)=\beta \frac{J_{2}}{2} \bar{\xi}_{2 s}(3), \\
& \bar{\gamma}_{3}(3)=\beta\left[\left(\frac{J_{3}}{2}+8 \frac{\psi_{6}^{2}}{v} s_{66}^{E 0}\right) \bar{\xi}_{3 s}(3)-2 \psi_{6} s_{66}^{E 0} \sigma_{6}+\bar{\mu}_{3} E_{3}\right], \quad \bar{\delta}(3)=\beta\left(\frac{J_{4}}{2} \bar{\sigma}_{s}(3)+\Delta\right) .
\end{aligned}
$$

Differentiating (3.15) with respect to the stress, we obtain coefficients of piezoelectric strain of Rochelle salt

$$
\begin{aligned}
& d_{i j p}\left(\sigma_{5}, E_{2}\right)=d_{14}^{0}-\frac{\bar{\mu}_{i}}{v} 2 s_{j j}^{E 0} \psi_{j} \beta F_{2 i p}\left(\bar{\xi}_{2 p}(2), \bar{\sigma}_{p}(2)\right), \quad(i=1,2), \\
& d_{i j p}\left(\sigma_{6}, E_{3}\right)=d_{14}^{0}-\frac{\bar{\mu}_{i}}{v} 2 s_{j j}^{E 0} \psi_{j} \beta F_{2 i p}\left(\bar{\xi}_{3 p}(3), \bar{\sigma}_{p}(3)\right), \quad(i=1,3), \\
& d_{i j s}\left(\sigma_{5}, E_{2}\right)=d_{14}^{0}-\frac{\bar{\mu}_{i}}{v} 2 s_{j j}^{E 0} \psi_{j} \beta F_{2 i s}\left(\bar{\xi}_{1 s}(2), \bar{\xi}_{2 s}(2), \bar{\xi}_{3 s}(2), \bar{\sigma}_{s}(2)\right), \quad(i=1,2), \\
& d_{i j s}\left(\sigma_{6}, E_{3}\right)=d_{14}^{0}-\frac{\bar{\mu}_{i}}{v} 2 s_{j j}^{E 0} \psi_{j} \beta F_{2 i s}\left(\bar{\xi}_{1 s}(3), \bar{\xi}_{2 s}(3), \bar{\xi}_{3 s}(3), \bar{\sigma}_{s}(3)\right), \quad(i=1,3) .
\end{aligned}
$$

From (3.16) we obtain expressions for the constant of piezoelectric strain

$$
g_{i j}=\left(\frac{\partial E_{i}}{\partial \sigma_{j}}\right)_{P_{i}}=\frac{d_{i j}}{\chi_{i i}^{\sigma}} .
$$

Differentiating (3.13) with respect to the stresses, we find the following relations for the compliances of Rochelle salt at a constant field

$$
\begin{aligned}
& s_{j j p}^{E}\left(\sigma_{5}, E_{2}\right)=s_{j j}^{E 0}+4 \frac{\psi_{j}^{2}}{v}\left(s_{j j}^{E 0}\right)^{2} \beta F_{2 i p}\left(\bar{\xi}_{2 p}(2), \bar{\sigma}_{p}(2)\right), \quad(i=1,2), \\
& s_{j j p}^{E}\left(\sigma_{6}, E_{3}\right)=s_{j j}^{E 0}+4 \frac{\psi_{j}^{2}}{v}\left(s_{j j}^{E 0}\right)^{2} \beta F_{2 i p}\left(\bar{\xi}_{3 p}(3), \bar{\sigma}_{p}(3)\right), \quad(i=1,3), \\
& s_{j j s}^{E}\left(\sigma_{5}, E_{2}\right)=s_{j j}^{E 0}+4 \frac{\psi_{j}^{2}}{v}\left(s_{j j}^{E 0}\right)^{2} \beta F_{2 i s}\left(\bar{\xi}_{1 s}(2), \bar{\xi}_{2 s}(2), \bar{\xi}_{3 s}(2), \bar{\sigma}_{s}(2)\right), \quad(i=1,2), \\
& s_{j j s}^{E}\left(\sigma_{6}, E_{3}\right)=s_{j j}^{E 0}+4 \frac{\psi_{j}^{2}}{v}\left(s_{j j}^{E 0}\right)^{2} \beta F_{2 i s}\left(\bar{\xi}_{1 s}(3), \bar{\xi}_{2 s}(3), \bar{\xi}_{3 s}(3), \bar{\sigma}_{s}(3)\right), \quad(i=1,3) .
\end{aligned}
$$

The transition temperatures $T_{c 1}$ and $T_{c 2}$ are determined from the condition that the inverse static dielectric susceptibility of free crystal $\chi_{11}^{\sigma}(0)$ vanishes at $T \rightarrow T_{c 1}$ and $T \rightarrow T_{c 2}$.

\section{Discussion}

To calculate the temperature and field $E_{i}$ dependences of the dielectric, piezoelectric, elastic, and thermal characteristics of Rochelle salt we have to set the values of the following parameters: interaction constants $J, K_{12}, K_{13}, K_{14}$; parameter $\Delta$; deformation potentials $\psi_{j}$; effective dipole moments $\mu_{i}$; and the "seed" dielectric susceptibilties $\chi_{22}^{\varepsilon 0}, \chi_{33}^{\varepsilon 0}$, coefficients of piezoelectric stress $e_{i j}^{0}$, elastic constants $c_{j j}^{E 0}$.

The unit cell volume is a linear function of temperature, since the lattice constants of Rochelle salt are also almost linear functions of temperature [38,39]. The volume thermal expansion coefficient, according to $[38,39]$ equals $0.00014 \mathrm{~cm}^{3} / \mathrm{K}$ and $0.00013 \mathrm{~cm}^{3} / \mathrm{K}$, respectively. Using the data of [39], we find the temperature dependence of the unit cell volulme of Rochelle salt

$$
v=1.0438[1+0.00013(T-190)] \cdot 10^{-21} \mathrm{~cm}^{3} .
$$

In order to determine the values of $J, K, \Delta$ and $\psi_{4}$ in [27] a line on the $(a, b)$ phase diagram was found, where

$$
a=\frac{K-J}{K+J+\frac{8}{v} \psi_{4}^{2} s_{44}^{E 0}}, \quad b=\frac{8 \Delta}{K+J+\frac{8}{v} \psi_{4}^{2} s_{44}^{E 0}} .
$$


For the points of this line, two second order phase transitions take place at $T_{c 1}=255.02 \mathrm{~K}$ and $T_{c 2}=296.86 \mathrm{~K}$. With $a$ and $b$ decreasing along this line, the maximal value of $\xi_{1}$ increases. The terminal point of this line was chosen $(a=0.295, b=0.648)$, that is, such values of $a$ and $b$, when the value of $\xi_{1}$ is maximal. Hence, the following values of the parameters were determined $\left.\left(J+K_{12}\right) / k_{\mathrm{B}}=797.36 \mathrm{~K}, K_{13}+K_{14}\right) / k_{\mathrm{B}}=1468.83 \mathrm{~K}, \Delta / k_{\mathrm{B}}=737.33 \mathrm{~K}, \psi_{4} / k_{\mathrm{B}}=-760 \mathrm{~K}$. Also [27] the effective dipole moment was determined $\mu_{1}=[2.52+0.0066(T-190)] \cdot 10^{-18} \mathrm{esu} \cdot \mathrm{cm}$.

In [27] the temperature dependences of polarization $P_{1}$, inverse static dielectric susceptibilities of clamped and free crystals $\chi_{11}^{-1}$, elastic constant $c_{44}^{E}$, piezoelectric characteristics $e_{14}, d_{14}, h_{14}$, $g_{14}$, and the spin contribution to the molar specific heat $\Delta C^{\sigma}$ were found. At the chosen values of the theory parameters, the theoretical results well agree with experimental data.

In this paper, we find the values of $J, K_{12}, K_{13}, K_{14}, \mu_{2}$, and $\mu_{3}$ by fitting the theoretical curves for $\varepsilon_{22}^{\varepsilon}$ and $\varepsilon_{33}^{\varepsilon}$ to experimental points of [41]. The accepted values of the parameters are given in the table 1 .

Table 1. The optimal set of the model parameters for the Rochelle salt crystals.

\begin{tabular}{|c|c|c|c|c|c|c|c|c|c|}
\hline & $J / k_{\mathrm{B}}$ & $K_{12} / k_{\mathrm{B}}$ & $K_{13} / k_{\mathrm{B}}$ & $K_{14} / k_{\mathrm{B}}$ & $\Delta / k_{\mathrm{B}}$ & $\mu_{20}$ & $\mu_{21}$ & $\mu_{30}$ & $\mu_{31}$ \\
& $\mathrm{~K}$ & $\mathrm{~K}$ & $\mathrm{~K}$ & $\mathrm{~K}$ & $\mathrm{~K}$ & & & & \\
\hline 1 & 247.36 & 550 & 400 & 1068.83 & 737.33 & 6.5 & 0.0065 & 8.67 & 0.0115 \\
\hline
\end{tabular}

At these values of the parameters, we have $\tilde{J}_{1}=2266.19 \mathrm{~K}, \tilde{J}_{2}=366.19 \mathrm{~K}, \tilde{J}_{3}=-971.47 \mathrm{~K}$, $\tilde{J}_{4}=-671,47 \mathrm{~K}$. The effective dipole moments $\mu_{2}$ and $\mu_{3}$ are taken as linear functions of temperature $\mu_{2}=\left[\mu_{20}+\mu_{21}(T-298)\right] \cdot 10^{-18} \mathrm{esu} \mathrm{cm}, \mu_{3}=\left[\mu_{30}+\mu_{31}(T-298)\right] \cdot 10^{-18} \mathrm{esu} \mathrm{cm}$.

The "seed" parameters are taken to be equal to $\chi_{22}^{\varepsilon 0}=0.05, \chi_{33}^{\varepsilon 0}=0.05, e_{25}^{0}=-0.2 \cdot 10^{4} \mathrm{esu} / \mathrm{dyn}$, $e_{36}^{0}=0.2 \cdot 10^{4} \mathrm{esu} / \mathrm{dyn}, c_{55}^{E 0}=3.6 \cdot 10^{10} \mathrm{dyn} / \mathrm{cm}^{2}, c_{66}^{E 0}=10 \cdot 10^{10} \mathrm{dyn} / \mathrm{cm}^{2}$.

The deformation parameters $\psi_{5}$ and $\psi_{6}$ are chosen by fitting all theoretical piezoelectric coefficients to experimental points given at $T=298 \mathrm{~K}$ in [42]. As a result, $\psi_{5} / k_{\mathrm{B}}=1650 \mathrm{~K}, \psi_{6} / k_{\mathrm{B}}=-1550 \mathrm{~K}$.

Let us now explore the calculated temperature curves of the physical characteristics of Rochelle salt at different values of the electric fields $E_{2}$ and $E_{3}$. We should mention that the calculations are performed at the fields up to $50 \mathrm{MV} / \mathrm{m}$. Obviously, the experimental measurements can be performed at much lower fields only, because so high fields can destroy the samples.

The temperature dependences of the mean values of pseudospins at $E_{2}=E_{3}=0$ and in fields $E_{2}$ or $E_{3}$ are shown in figure 2 . In the absence of the field $\eta_{1}=\eta_{2},-\eta_{3}=-\eta_{4}$ in the ferroelectric phase, and $\eta_{1}=\eta_{2}=-\eta_{3}=-\eta_{4}$ in the paraelectric phases. The electric field $E_{2}$ splits the values of pseudospins in the ferroelectric phase and narrows its temperature range, whereas in the paraelectric phases $\eta_{1}=-\eta_{3}, \eta_{2}=-\eta_{4}$. The field $E_{3}$ also splits the values of pseudospins in the ferroelectric phase, but widens its temperature range, and $\eta_{1}=-\eta_{4}, \eta_{2}=-\eta_{3}$ in the paraelectric phase.

The effect of electric fields $E_{2}$ and $E_{3}$ on the projections of the dipole moments is shown in figures 3 and 4 .

In figure 5 we show the field $E_{2}$ and $E_{3}$ dependences of the phase transition temperatures $T_{c 1}$ and $T_{c 2}$. With increasing $E_{2}$, the ferroelectric phase narrows and disappears and $E_{2} \sim 30 \mathrm{MV} / \mathrm{m}$. There is no direct experimental evidence for this dependence so far, but the results of [47], where the relaxation phenomena in Rochelle salt were explored experimentally in a transverse electric field, can be considered as a certain indirect confirmation of this. The field $E_{3}$, on the contrary, widens the temperature range of the ferroelectric phase.

Dependences of the transition temperatures $T_{c 1}$ and $T_{c 2}$ on the fields $E_{2}$ and $E_{3}$ up to $5 \mathrm{MV} / \mathrm{m}$ and on squares of the fields are given in figures 6 and 7 , respectively.

As one can see, the dependences $T_{c 1}\left(E_{2}\right)$ and $T_{c 2}\left(E_{3}\right)$ are quadratic and at fields below $5 \mathrm{MV} / \mathrm{m}$ can be expressed as

$$
\begin{array}{ll}
T_{c 1}\left(E_{2}\right)=T_{c 1}+k_{12} E_{2}^{2}, & T_{c 2}\left(E_{2}\right)=T_{c 2}+k_{22} E_{2}^{2}, \\
T_{c 1}\left(E_{3}\right)=T_{c 1}+k_{13} E_{3}^{2}, & T_{c 2}\left(E_{3}\right)=T_{c 2}+k_{23} E_{3}^{2},
\end{array}
$$



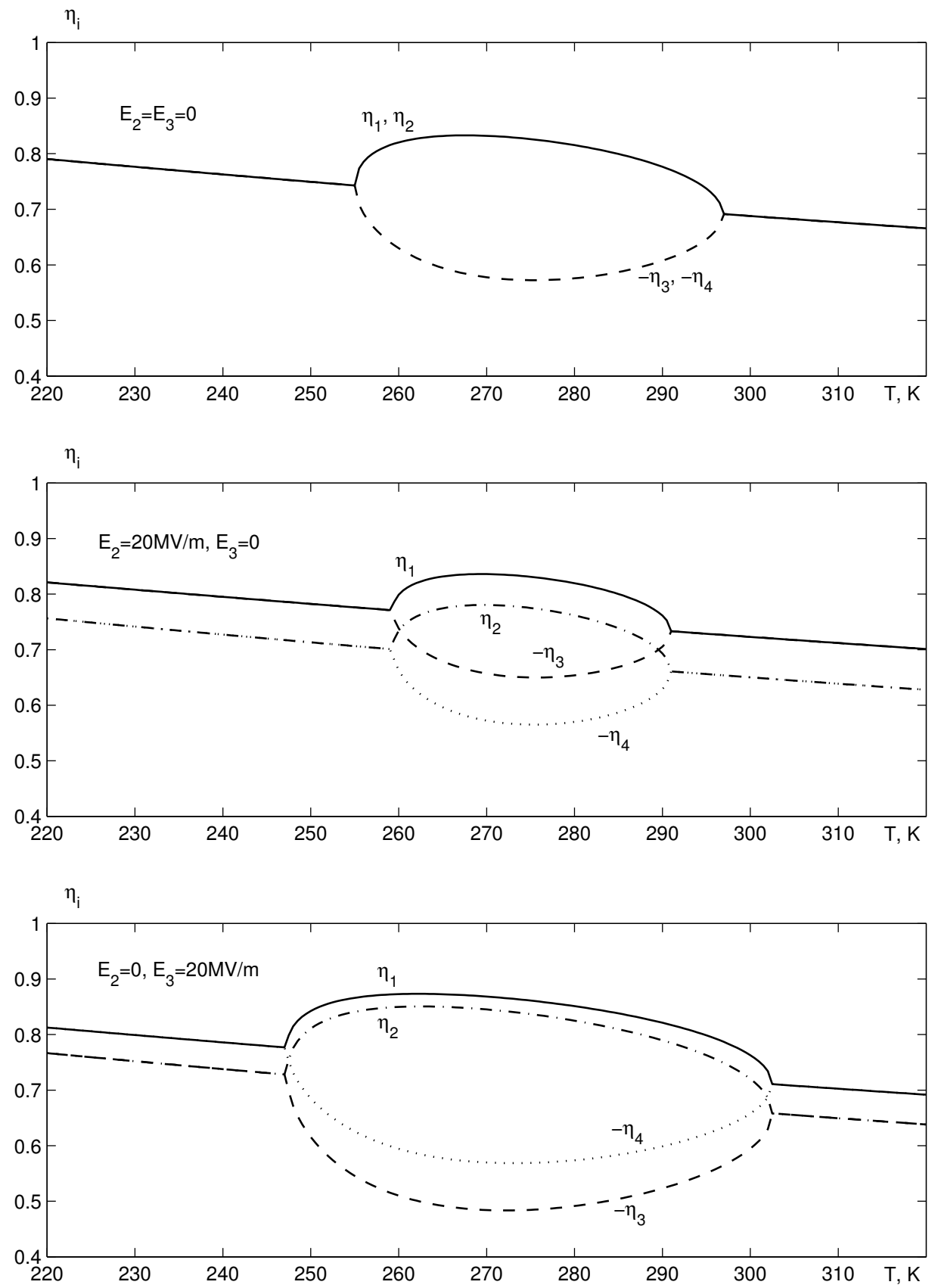

Figure 2. The temperature dependence of the mean values of pseudospins $\eta_{1}, \eta_{2}, \eta_{3}, \eta_{4}$ in transverse fields.

where $k_{12}=0.009 \frac{\mathrm{Km}^{2}}{\mathrm{MV}^{2}}, k_{22}=-0.013 \frac{\mathrm{Km}^{2}}{\mathrm{MV}^{2}}, k_{13}=-0.021 \frac{\mathrm{Km}^{2}}{\mathrm{MV}^{2}}, k_{23}=0.016 \frac{\mathrm{Km}^{2}}{\mathrm{MV}^{2}}$.

In figure 8 we plot the temperature dependences of polarization components $P_{i}$ at different values of the fields $E_{2}$ and $E_{3}$. With increasing field $E_{2}$ the spontaneous polarization $P_{1}$ decreases (this is observed experimentally) and the polarization $P_{3}$ induced by the field $E_{2}$ increases. The latter polarization is negative and by one order of magnitude smaller than $P_{1}$. The polarization $P_{2}$ induced by the field $E_{2}$ has no peculiarities and is practically temperature independent at low fields. An increase in the field $E_{3}$ increases $P_{1}$ as well as the polarization $P_{2}$ induced by this field; 

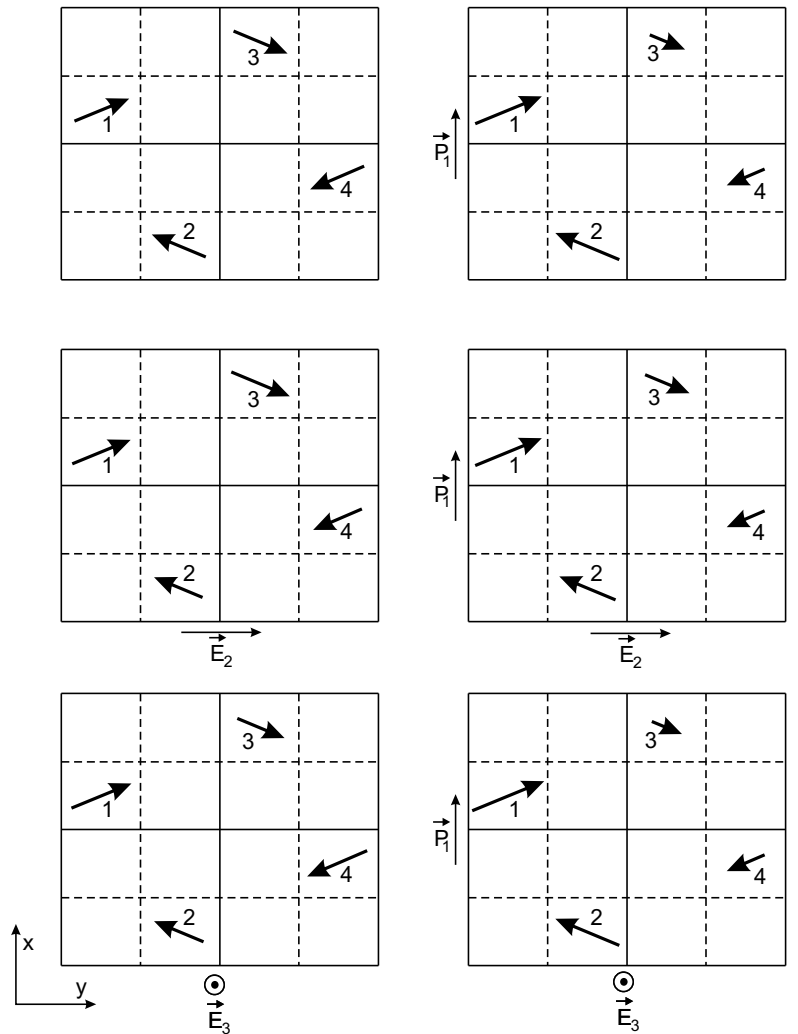

Figure 3. Projections of the dipole moments on the XY plane in the paraelectric (left) and ferroelectric (right) phases.
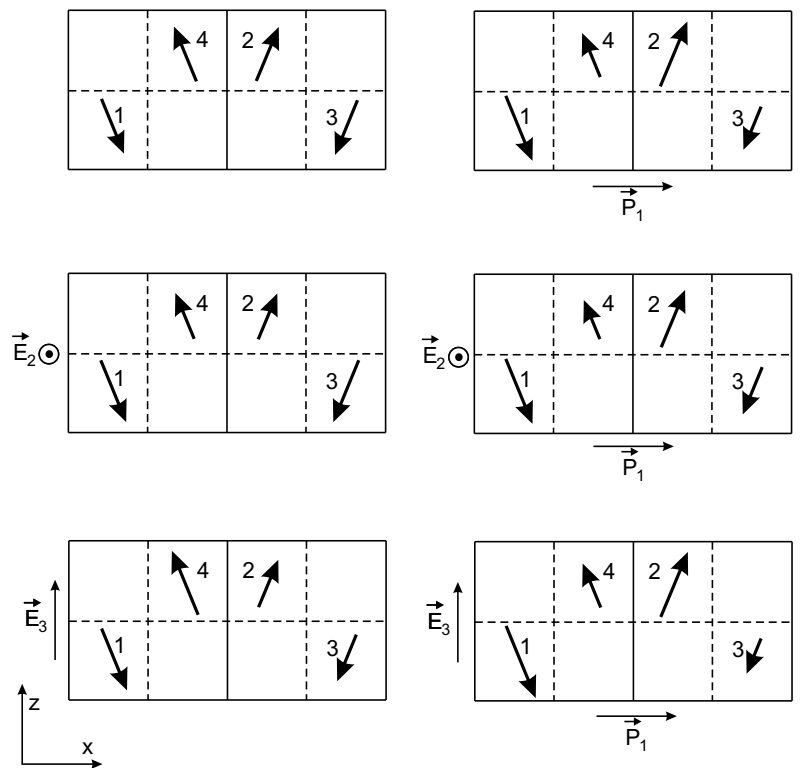

Figure 4. Projections of the dipole moments on the $\mathrm{XZ}$ plane in the paraelectric (left) and ferroelectric (right) phases. 


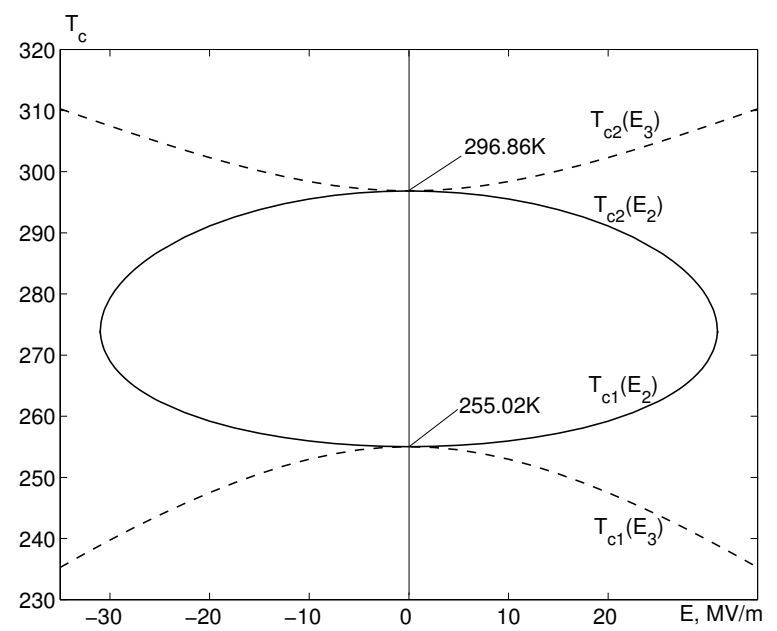

Figure 5. Dependence of the lower $\left(T_{c 1}\right)$ and upper $\left(T_{c 2}\right)$ Curie temperatures on the fields $E_{2}$ and $E_{3}$.
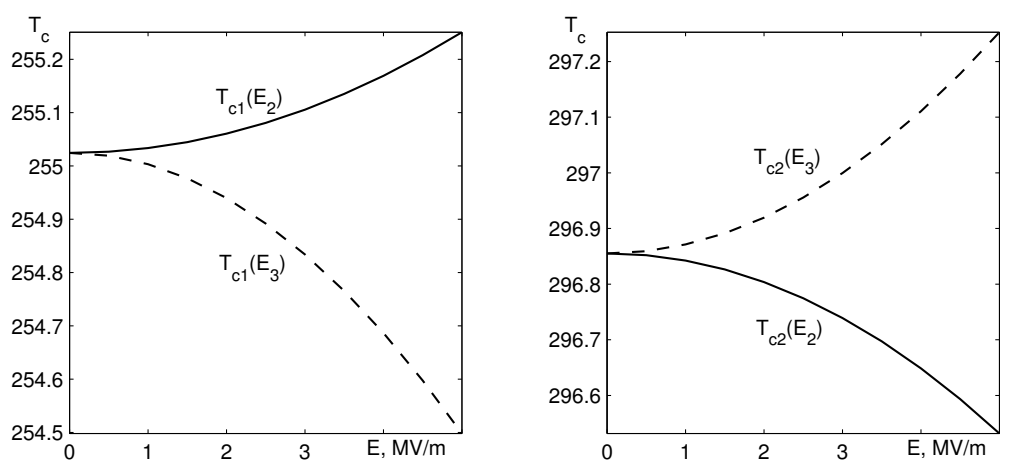

Figure 6. Dependence of the lower $\left(T_{c 1}\right)$ and upper $\left(T_{c 2}\right)$ Curie temperatures on the fields $E_{2}$ and $E_{3}$.
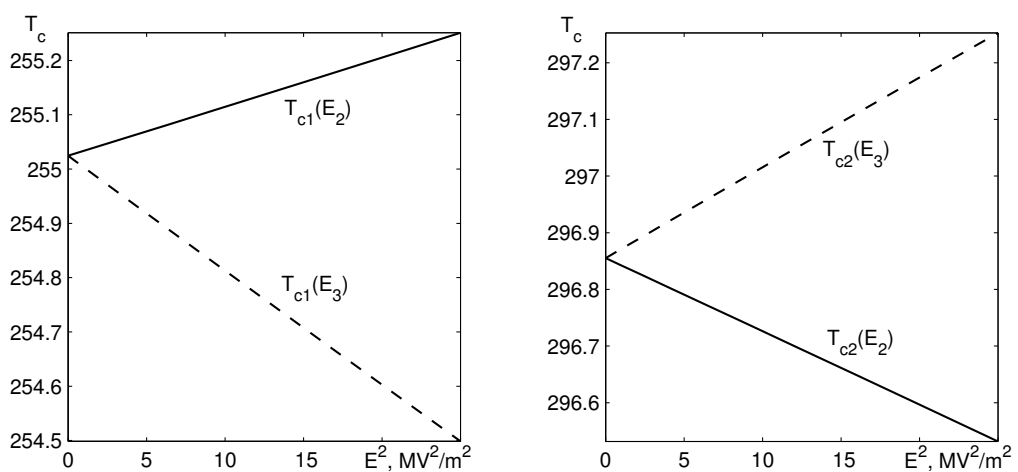

Figure 7. Dependence of the lower $\left(T_{c 1}\right)$ and upper $\left(T_{c 2}\right)$ Curie temperatures on the squares of the fields $E_{2}^{2}$ and $E_{3}^{2}$. 

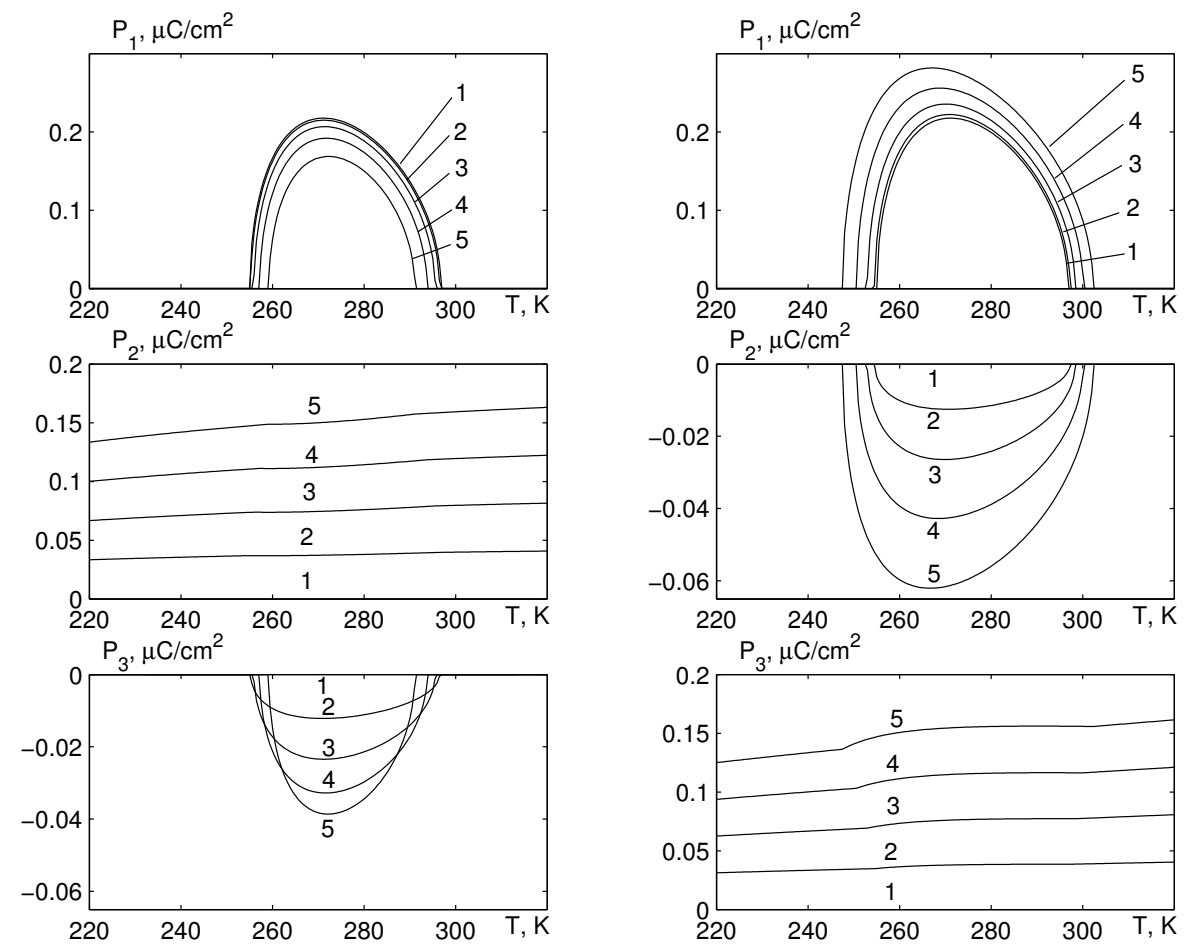

Figure 8. The temperature dependence of polarization components in the field $E_{2}$ (left) and $E_{3}$ (right) $(\mathrm{kV} / \mathrm{cm}): 1-0 ; 2-50 ; 3-100 ; 4-150 ; 5-200$.

this increase is much faster than of $P_{3}\left(E_{2}\right)$. The shape of the polarization $P_{3}\left(E_{3}\right)$ induced by the field $E_{3}$ is similar to $P_{2}\left(E_{2}\right)$, and the magnitude of $P_{3}\left(E_{3}\right)$ is almost equal to that of $P_{2}\left(E_{2}\right)$.

Temperature behavior of the strains $\varepsilon_{4}, \varepsilon_{5}, \varepsilon_{4}$ under the field $E_{2}$ or $E_{3}$ is similar (almost proportional) to the temperature behavior of polarizations $P_{1},-P_{2}, P_{3}$ respectively [49].

Changes in the temperature curves of the inverse components of static dielectric susceptibilities of mechanically clamped and free crystals of Rochelle salt induced by transverse electric field $E_{2}$ are shown in figure 9 . The values of $\chi_{11}^{\varepsilon}\left(E_{2}\right)^{-1}, \chi_{11}^{\sigma}\left(E_{2}\right)^{-1}$ decrease with increasing field in the ferroelectric phase and increase in the paraelectric phase. The field $E_{3}$ has an opposite effect [49]. In the temperature curves of $\chi_{33}^{\varepsilon}\left(E_{2}\right)^{-1}$ and $\chi_{22}^{\varepsilon}\left(E_{3}\right)^{-1}$ there arise minima at the transition points, deepening with increasing fields. The values of $\chi_{33}^{\sigma}\left(E_{2}\right)^{-1}$ and $\chi_{22}^{\sigma}\left(E_{3}\right)^{-1}$ decrease with increasing fields and turn to zero at the transition points. The values of $\chi_{22}^{\varepsilon}\left(E_{2}\right)^{-1}, \chi_{22}^{\sigma}\left(E_{2}\right)^{-1}, \chi_{33}^{\varepsilon}\left(E_{3}\right)^{-1}$, $\chi_{33}^{\sigma}\left(E_{3}\right)^{-1}$ do not depend on the fields in the paraelectric phases and decrease with the fields in the ferroelectric phase.

The inverse susceptibilities $\chi_{22}^{\sigma}\left(E_{2}\right)^{-1}$ and $\chi_{33}^{\sigma}\left(E_{3}\right)^{-1}$ have jumps at the transition temperatures, the jump magnitudes increasing with the fields.

The temperature dependence of inverse static permittivities of mechanically free crystal of Rochelle salt $\left(\varepsilon_{22}^{\sigma}\right)^{-1}$ in the field $E_{2}$ below $5 \mathrm{MV} / \mathrm{m}$ is shown in figure 10 , and that of $\left(\varepsilon_{33}^{\sigma}\right)^{-1}$ in the field $E_{3}$ below $5 \mathrm{MV} / \mathrm{m}$ is shown in figure 11. Jumps of $\Delta\left(\varepsilon_{22}^{\sigma}\right)^{-1}$ and $\Delta\left(\varepsilon_{33}^{\sigma}\right)^{-1}$ at the transition points are proportional to the squares of the fields (figure 12), and

$$
\begin{array}{ll}
\Delta\left(\varepsilon_{22}^{\sigma(1)}\right)^{-1}=\bar{k}_{12} E_{2}^{2}, & \Delta\left(\varepsilon_{22}^{\sigma(2)}\right)^{-1}=\bar{k}_{22} E_{2}^{2}, \\
\Delta\left(\varepsilon_{33}^{\sigma(1)}\right)^{-1}=\bar{k}_{13} E_{3}^{2}, & \Delta\left(\varepsilon_{33}^{\sigma(2)}\right)^{-1}=\bar{k}_{23} E_{3}^{2},
\end{array}
$$

where $\bar{k}_{12}=0.068 \frac{\mathrm{m}^{2}}{\mathrm{MV}^{2}}, \bar{k}_{22}=0.048 \frac{\mathrm{m}^{2}}{\mathrm{MV}^{2}}, \bar{k}_{13}=0.440 \frac{\mathrm{m}^{2}}{\mathrm{MV}^{2}}, \bar{k}_{23}=0.080 \frac{\mathrm{m}^{2}}{\mathrm{MV}^{2}}$.

The temperature dependences of $c_{44}^{E}, s_{44}^{E}, e_{14}, d_{14}, h_{14}$, and $g_{14}$ at different values of the field $E_{2}$ are shown in figure 13. The elastic constat $c_{44}^{E}$ increases with the field $E_{2}$ in the paraelectric phases 

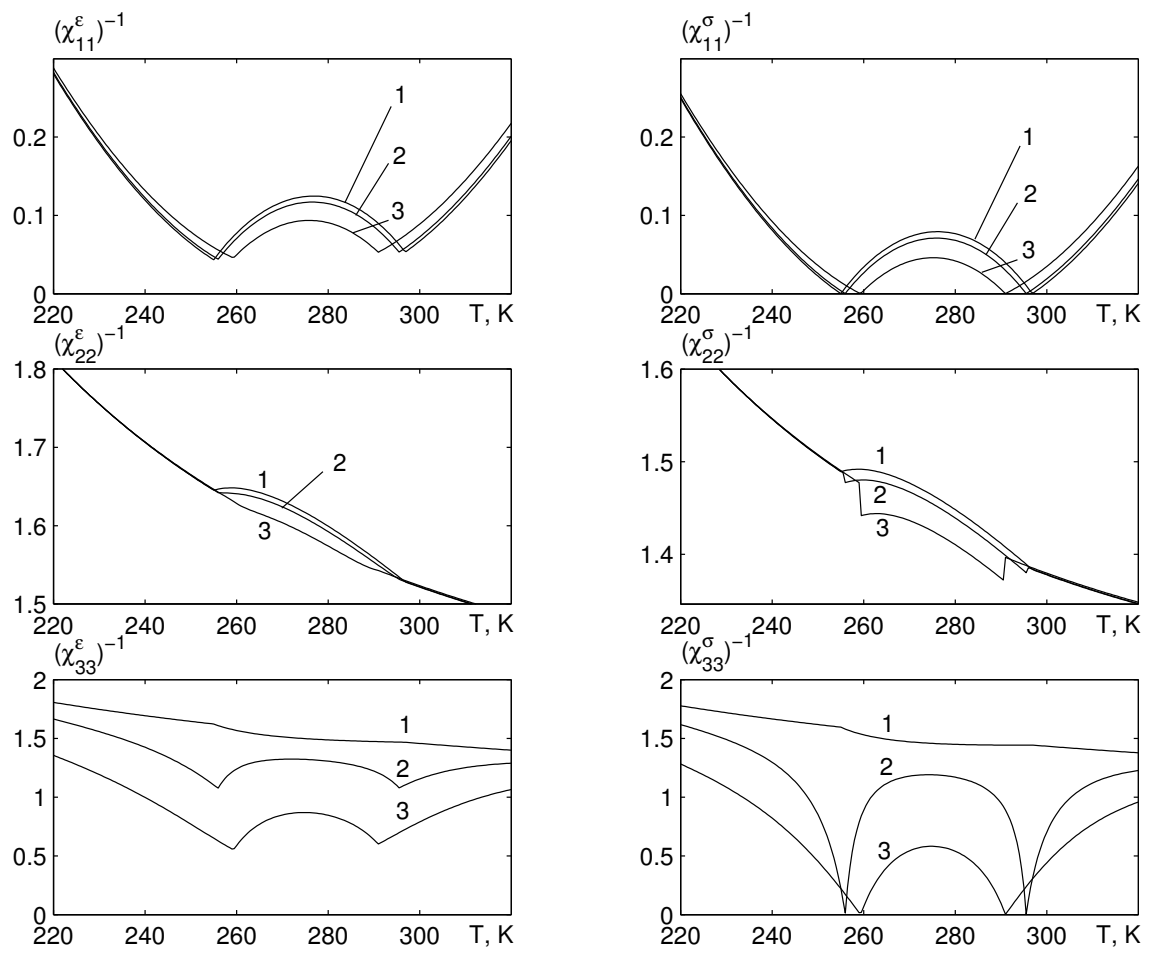

Figure 9. The temperature dependence of inverse static susceptibilities of clamped and free crystals: $\mathrm{a}-\left(\chi_{11}^{\varepsilon}\right)^{-1},\left(\chi_{11}^{\sigma}\right)^{-1} ; \mathrm{b}-\left(\chi_{22}^{\varepsilon}\right)^{-1},\left(\chi_{22}^{\sigma}\right)^{-1} ; \mathrm{c}-\left(\chi_{33}^{\varepsilon}\right)^{-1},\left(\chi_{33}^{\sigma}\right)^{-1}$ at different values of the field $E_{2}(\mathrm{kV} / \mathrm{cm}): 1-0 ; 2-100 ; 3-200$.
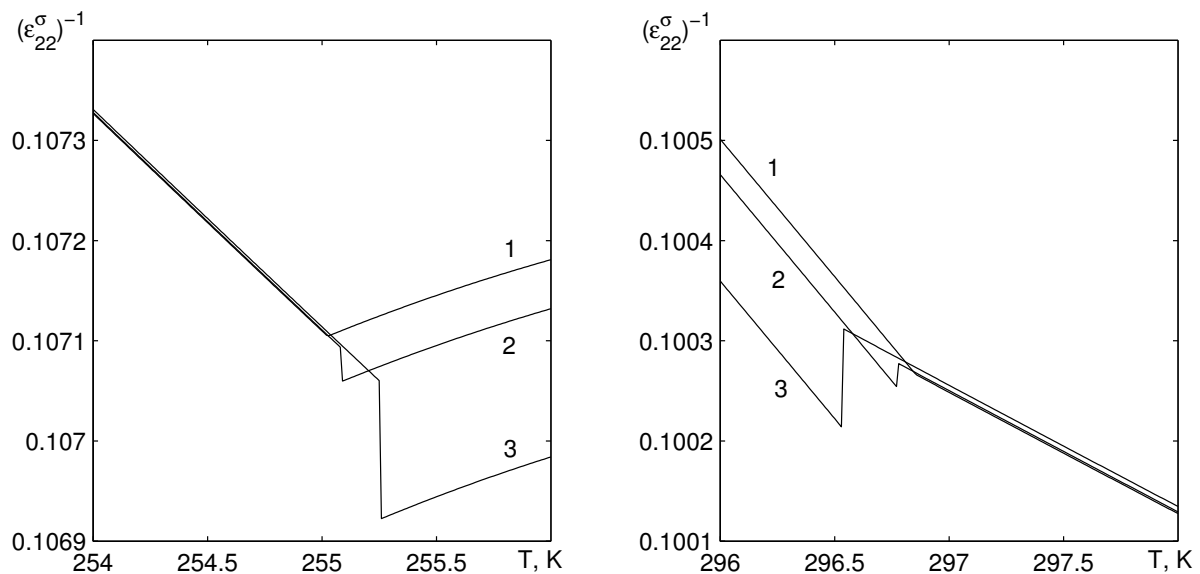

Figure 10. The temperature dependence of inverse static permittivity $\left(\varepsilon_{22}^{\sigma}\right)^{-1}$ of a free crystal near the lower (left) and upper (right) transition points at different values of the electric field $E_{2}(\mathrm{MV} / \mathrm{m}): 1-0 ; 2-2.5 ; 3-5$.

and decreases in the ferroelectric phase. The field $E_{3}$ has an opposite effect [49]. An increase in the field $E_{2}$ increases the compliance $s_{44}^{E}$ and all piezoelectric coefficients in the ferroelectric phase and decreases them in the paraelectric phases. The field $E_{3}$ has an opposite effect. The effect of the fields $E_{2}$ and $E_{3}$ on the values of $h_{14}$ and $g_{14}$ is very small.

The changes in the temperature curves of $c_{55}^{E}, s_{55}^{E}, e_{25}, d_{25}, h_{25}$, and $g_{25}$ with increasing fi- 

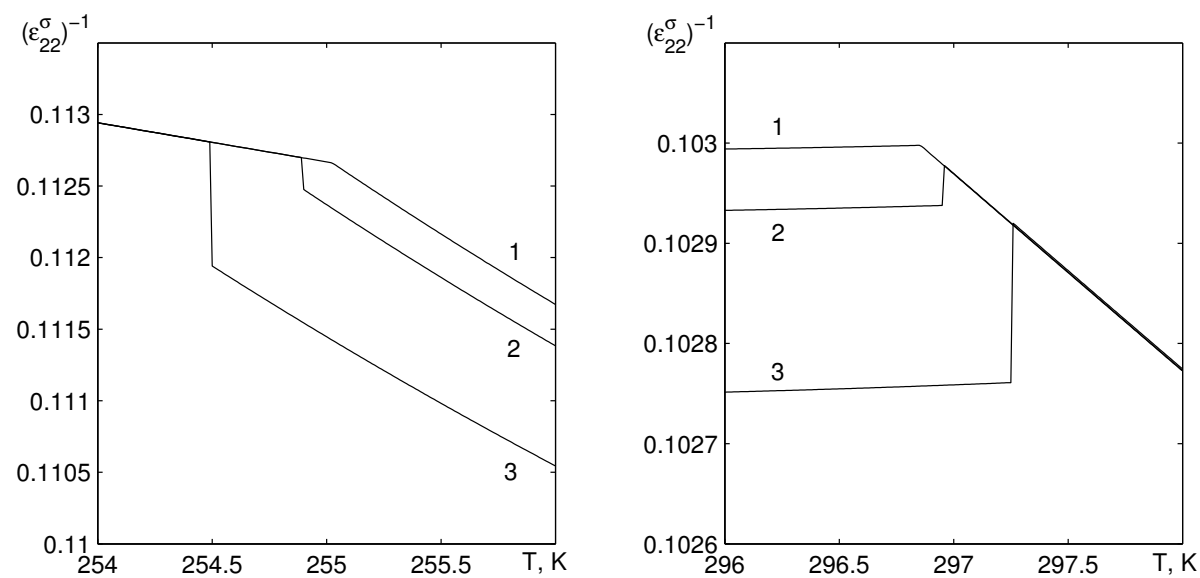

Figure 11. The temperature dependence of inverse static permittivity $\left(\varepsilon_{33}^{\sigma}\right)^{-1}$ of a free crystal near the lower (left) and upper (right) transition points at different values of the electric field $E_{3}(\mathrm{Mv} / \mathrm{m}): 1-0 ; 2-2.5 ; 3-5$.
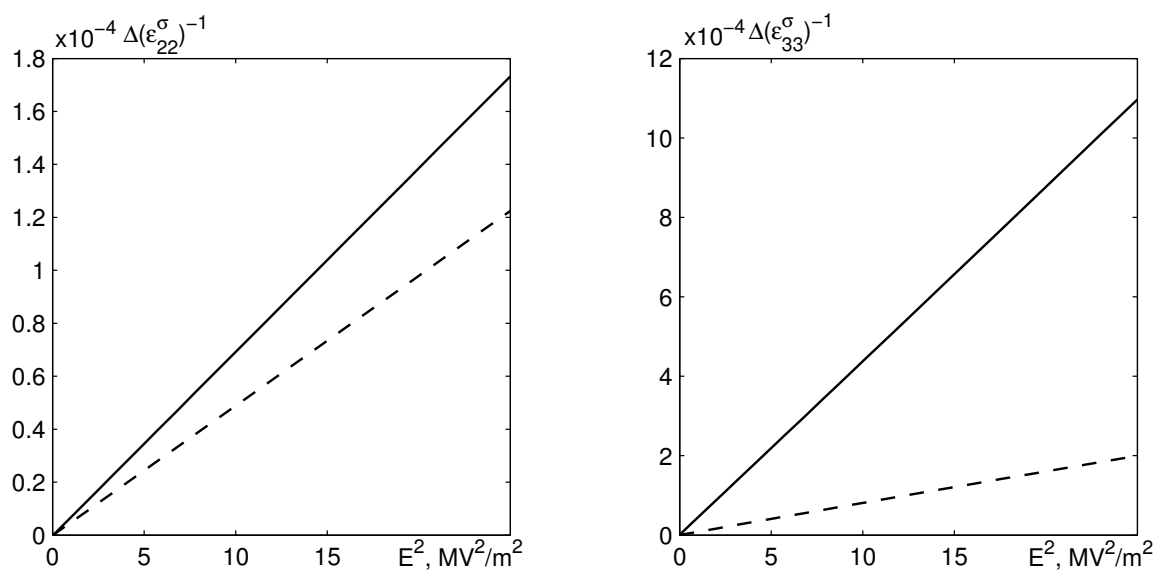

Figure 12. Dependences of the jumps of inverse dielectric permittivity $\left(\varepsilon_{22}^{\sigma}\right)^{-1}$ on the square of the field $E_{2}$ and of $\left(\varepsilon_{33}^{\sigma}\right)^{-1}$ on the square of the field $E_{3}$ near the lower (left) and upper (right) transition points.

eld $E_{2}$ are shown in figure 14, whereas the temperature curves of $c_{66}^{E}, s_{66}^{E}, e_{36}, d_{36}, h_{36}, g_{36}$ at different values of $E_{2}$ are given in figure 15. These characteristics with increasing field $E_{3}$ are shown in [49]. The elastic constants $c_{55}^{E}\left(E_{2}\right)$ and $c_{66}^{E}\left(E_{3}\right)$ slightly decrease with fields in the ferroelectric phase and are field independent in the paraelectric phase. Within the increasing fields in the temperature curves of $c_{55}^{E}\left(E_{3}\right)$ and $c_{66}^{E}\left(E_{2}\right)$, the minima arise. The piezoelectric coefficients $e_{25}\left(E_{2}\right), e_{36}\left(E_{3}\right), d_{25}\left(E_{2}\right), d_{36}\left(E_{3}\right)$ are field independent in the paraelectric phases, whereas $h_{25}\left(E_{2}\right), h_{36}\left(E_{3}\right), g_{25}\left(E_{2}\right), g_{36}\left(E_{3}\right)$ are field independent at all temperatures. In all other cases, an increasing field $E_{2}$ or $E_{3}$ increases the piezoelectric coefficients. In the temperature curves of $e_{25}\left(E_{3}\right), e_{36}\left(E_{2}\right), d_{25}\left(E_{3}\right), d_{36}\left(E_{2}\right)$, maxima arise at the transition temperatures, increasing with the fields. Small maxima at the transition points are also induced in the temperature dependences of $h_{25}\left(E_{3}\right), h_{36}\left(E_{2}\right), g_{25}\left(E_{3}\right), g_{36}\left(E_{2}\right)$. Finally, jumps of $d_{25}\left(E_{2}\right)$ and $d_{36}\left(E_{3}\right)$ are observed at the transitions, with the jump values $\Delta d_{36}\left(E_{3}\right)$ being proportional to $E_{2}^{2}$ and $E_{3}^{2}$, respectively.

The farther is temperature from $T_{c 1}$ and $T_{c 2}$, the higher is the field $E_{2}$ or $E_{3}$ at which the transition takes place, and the larger are the jumps $\Delta \varepsilon_{22}^{\sigma}\left(E_{2}\right)$ and $\Delta \varepsilon_{33}^{\sigma}\left(E_{3}\right)$. 

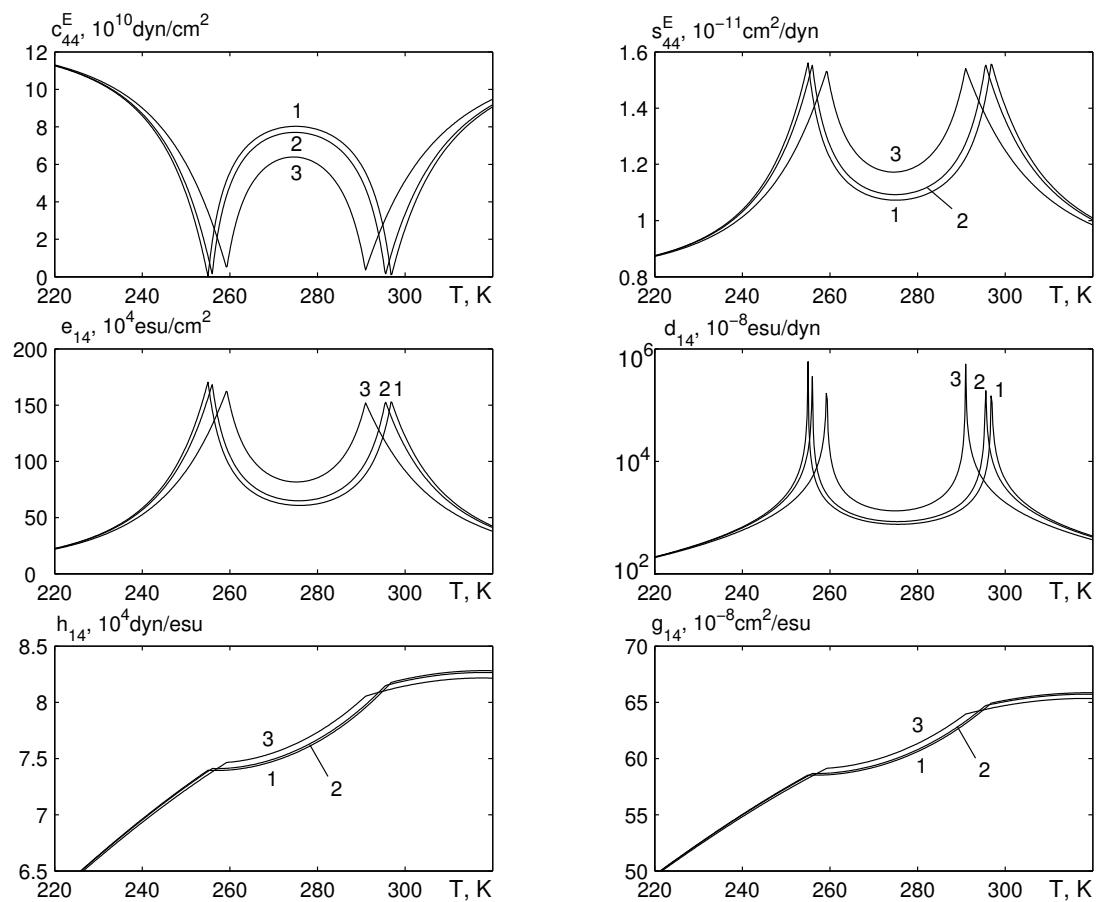

Figure 13. The temperature dependences of $c_{44}^{E}, s_{44}^{E}, e_{14}, d_{14}, h_{14}, g_{14}$ at different values of the field $E_{2}(\mathrm{kV} / \mathrm{cm}): 1-0 ; 2-100 ; 3-200$.
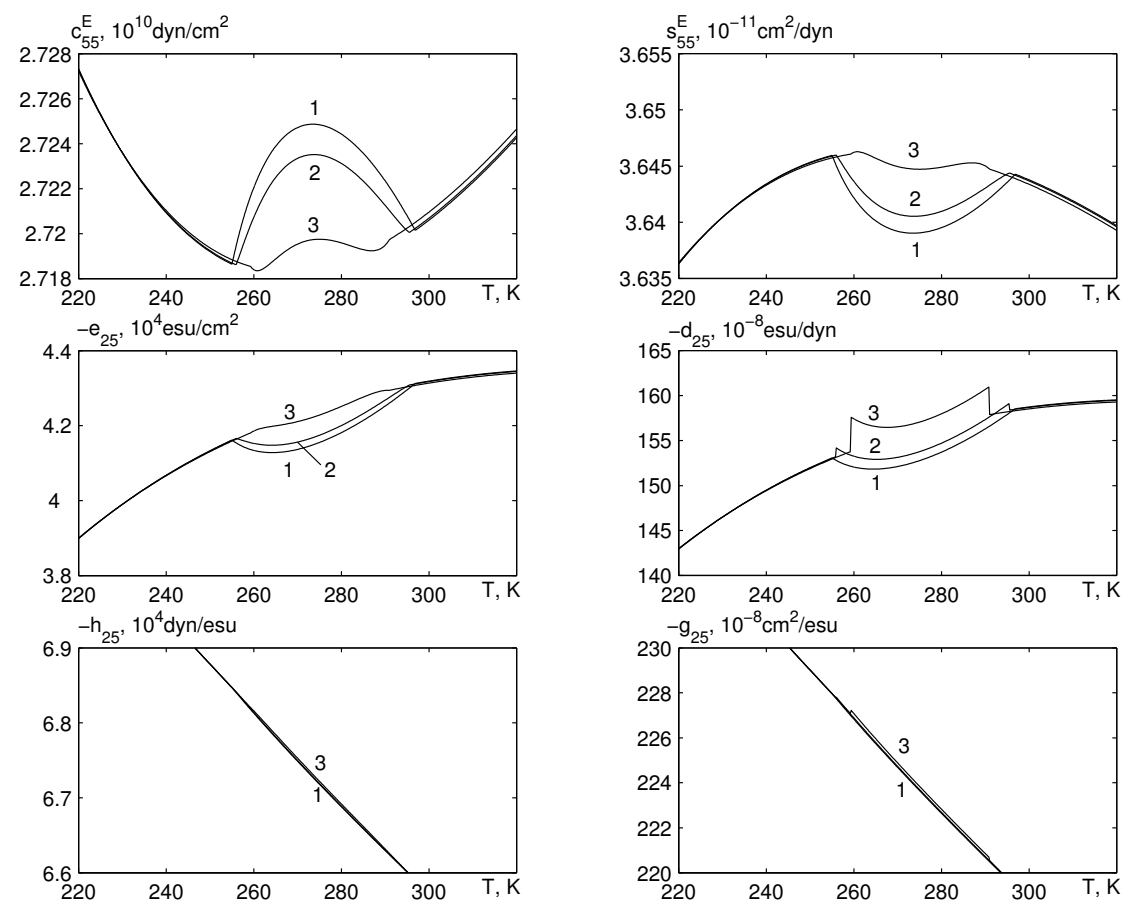

Figure 14. The temperature dependences of $c_{55}^{E}, s_{55}^{E}, e_{25}, d_{25}, h_{25}, g_{25}$ at different values of the electric field $E_{2}(\mathrm{kV} / \mathrm{cm}): 1-0 ; 2-100 ; 3-200$. 

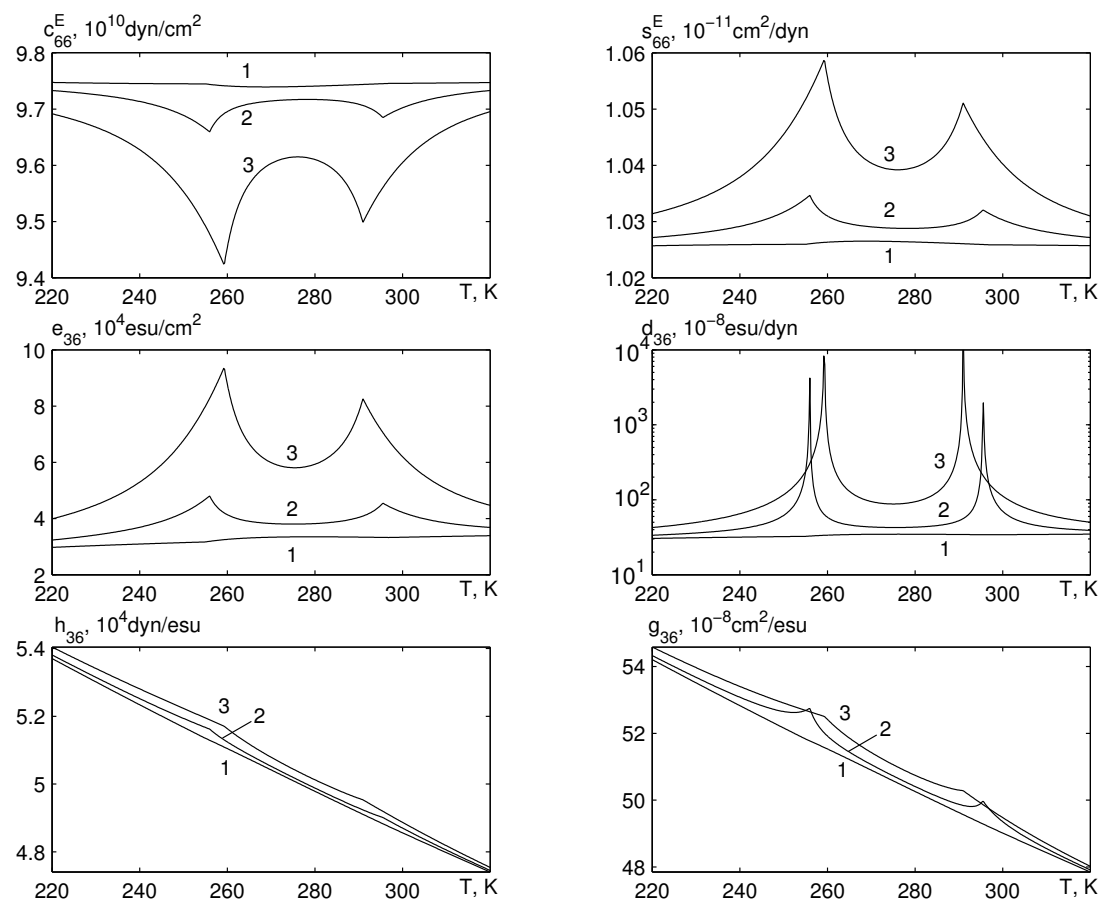

Figure 15. The temperature dependences of $c_{66}^{E}, s_{66}^{E}, e_{36}, d_{36}, h_{36}, g_{36}$ at different values of the electric field $E_{2}(\mathrm{kV} / \mathrm{cm}): 1-0 ; 2-100 ; 3-200$.

\section{Concluding remarks}

Within the framework of the four-sublattice pseudospin model [36] with piezoelectric coupling with the shear strains $\varepsilon_{4}, \varepsilon_{5}, \varepsilon_{6}$ in the mean field approximation we find the thermodynamic potential and Gibbs' function of the system. Therefrom we derive expressions for spontaneous polarization, components of the static dielectric permittivity tensors of mechanically free and clamped crystals, piezoelectric characteristics, and elastic constants. The proposed model at the proper choice of the theory parameters permits a good quantitative description of the available experimental data for Rochelle salt $[40-46,49]$. In contrast to the analogous longitudinal characteristics [27], the values of the transverse characteristics of Rochelle salt are much smaller and practically do not change at the phase transitions.

A thorough investigation of the effect of the transverse field $\left(E_{2}\right.$ and $\left.E_{3}\right)$ on the phase transitions and on physical properties of Rochelle salt is performed for the fist time. With increasing $E_{2}$ the temperature range of the ferroelectric phase narrows and disappears at $E_{2} \geqslant \tilde{E}_{2}$. On the contrary, the field $E_{3}$ widens the ferroelectric phase. Dependences of the transition temperatures $T_{c 1}$ and $T_{c 2}$ on the fields $E_{2}$ and $E_{3}$ are quadratic. The spontaneous polarization $P_{s}$ and strain $\varepsilon_{4}$ decrease with an increasing field $E_{2}$ (which qualitatively agrees with the experimental results [47]) and increase with the field $E_{3}$.

With an increasing field $E_{2}$, the values of the inverse susceptibilities $\left(\chi_{11}^{\varepsilon, \sigma}\right)^{-1}$ decrease in the ferroelectric phase and increase in the paraelectric phases. With an increasing $E_{2},\left(\chi_{22}^{\varepsilon, \sigma}\right)^{-1}$ decreases in the ferroelectric phase and is field independent in the paraelectric phase.

In the temperature curve $\left(\chi_{33}^{\varepsilon}\right)^{-1}$ there arise minima, gradually deepening with an increasing $E_{2}$. With an increasing $E_{3}$, the values of $\left(\chi_{33}^{\varepsilon, \sigma}\right)^{-1}$ increase in the ferroelectric phase and decrease in the paraelectric phases.

In the temperature curves of $\left(\chi_{22}^{\varepsilon}\right)^{-1}$ there also arise minima, deepening at an increasing $E_{3}$. In the paraelectric phase, $\left(\chi_{33}^{\varepsilon, \sigma}\right)^{-1}$ are independent of the field $E_{3}$, and decrease with the field in the ferroelectric phase. The values of the jumps of $\left(\varepsilon_{22}^{\sigma}\right)^{-1}$ and $\left(\varepsilon_{33}^{\sigma}\right)^{-1}$ at the phase transitions are 
proportional to the squares of the fields. Analogous results were obtained in $[48,50]$ in their studies of the transverse electric field dependence of the transverse dielectric susceptibility of glycinium phosphite.

\section{References}

1. Iona F., Shirane D. Ferroelectric crystals, Moscow, 1965 (in Russian).

2. Smolenskij G.A and other. Ferroelectrics and antiferroelectrics, Leningrad, 1971 (in Russian).

3. Beevers C.A., Hughes P.W., Proc. Roy. Soc, 1941, 177, 251.

4. Frazer B.C., McKeown M., Pepinsky R., Phys. Rev., 1954, 94, 1435.

5. Sandy F., Jones R.V., Phys. Rev., 1968, 168, No. 2, 481.

6. Kamba S., Schaack G., Petzelt J., Phys. Rev. B, 1995, 51, No. 21, 14998.

7. Volkov A.A., Kozlov G.B., Kryukova E.B., Petzelt Ya., J. exp. theor. phys., 1986, 90, No. 1, 192 (in Russian).

8. Shiozaki Y., Shimizu K., Suzuki E., Nozaki R., J. Korean Phys. Soc., 1998, 32, No. 91, S192.

9. Hlinka J., Kulda J., Kamba S., Petzelt J., Phys. Rev. B. 2001, 63, No. 5, 052102.

10. Shiozaki Y., Shimizu K., Nozaki R., Ferroelectrics, 2001, 261, 239.

11. Noda N., Nozaki R., Shiozaki Y., Phys. Rev. B, 2000, 62, No. 18, 12040.

12. Suzuki E., Amano A., Nozaki R., Shiozaki Y., Ferroelectrics, 1994, 152, 385.

13. Iwata Y., Koyano N., Shibuya I., Annu. Repts. Res. React. Inst. Kyoto Univ., 1989, 22, 87-91.

14. Mitsui T., Phys. Rev., 1958, 111, No. 5, 1259.

15. Zeks B., Shukla G.G., Blinc R., Phys. Rev. B, 1971, No. 3, 2305.

16. Vaks V.G., Introduction into microscopic theory of ferroelectrics, Moscow, 1973 (in Russian).

17. Kalenik J., Acta Phys. Pol., 1975, A48, No. 3, 387.

18. Zeks B., Shukla G.G., Blinc R., J.Phys. C, 1972, 33, 67.

19. Glauber J., J. Math. Phys., 1963, 4, No. 2, 294.

20. Blinc R., Zeks B. Ferroelectrics and antiferroelectrics. Lattice dynamics. Moscow, 1975 (in Russian).

21. Levitskii R.R., Verkholyak T.M., Kutny I.V., Hil I.G. Preprint of the Institute for Condensed Matter Physics, ICMP-01-11U, Lviv, 2001 (in Ukrainian).

22. Levitskii R.R., Verkholyak T.M., Kutny I.V., Hil I.G. Preprint arXiv:cond-mat/0106351, 2001.

23. Dublenych Yu.I., Preprint of the Institute for Condensed Matter Physics, ICMP-02-15U, Lviv, 2002 (in Ukrainian).

24. Levitsky R.R., Zachek I.R., Varanitsky V.I., Ukr. J. Phys., 1980, 25, No. 11, 1766.

25. Levitsky R.R., Antonyak Yu.T., Zachek I.R., Ukr. J. Phys., 1981, 26, No. 11, 1835.

26. Antonyak Yu.T., Volkov A.A., Zachek I.R., Kozlov G.B., Lebedev S.P., Levitskii R.R. Preprint of the Lebedev Physical Institute, No. 59., Moscow, 1982 (in Russian).

27. Levitskii R.R., Zachek I.R., Verkholyak T.M., Moina A.P., Phys. Rev. B, 2003, 67, No. 17, 174112.

28. Levitskii R.R., Zachek I.R., Verkholyak T.M., Moina A.P., Condens. Matter Phys., 2003, 6, No. 2(34), 261.

29. Kozlov G.B., Kryukova E.B., Lebedev S.P., Sobyanin A.A., J. exp. theor. phys., 1988, 94, No. 8, 304.

30. Levitskii R.R., Zachek I.R., Moina A.P., Andrusyk A.Ya. Condens. Matter Phys., 2004, 7, No. 1(37), 111.

31. Moina A.P., Levitskii R.R., Zachek I.R., Phys. Rev. B, 2005, 71, 134108.

32. Levitskii R.R., Zachek I.R., Moina A.P., Verkholyak T.M., J. Phys. Study, 2003, 7, No. 1, 106.

33. Moina A.P., Slivka A.G., Kedyulich V.M., Phys. stat. sol. (b), 2007, 244, No. 7, 2641.

34. Levitskii R.R., Moina A.P., Andrusyk A.Ya., Slivka A.G., Kedyulich V.M., J. Phys. Study, 2008, 12, No. 2, 2603.

35. Levitskii R.R., Andrusyk A.Ya. Preprint of the Institute for Condensed Matter Physics, ICMP-05-13U, Lviv, 2005 (in Ukrainian).

36. Stasyuk I.V., Velychko O.V., Ferroelectrics, 2005, 316, 51.

37. Fugiel B., Physica B: Condensed Matter., 2003, 325, 256.

38. Vigness J., Phys. Rev., 1935, 48, 198.

39. Bronowska W.J., J. Appl. Crystallogr., 1981, 14, 203.

40. Habblutzel J., Helv. Phys. Acta., 1939, 12, 489.

41. Forsch K., Muser H.E., Z. Naturforsch., 1968, 23, 1231.

42. Mason W. Piezoelectric crystals and its application in ultraacoustics, Moskow, 1952 (in Russian).

43. Zheludev I.S. Physics of crystal dielectrics, Moscow, 1968 (in Russian).

44. Valasek J., Phys. Rev., 1922, 19, 478. 
45. Jona F., Helv. phys. acta., 1950, 23, 795.

46. Schmidt H., Ferroelectrics, 1976, 14, 575.

47. Kalisz L., Fugiel B., Ziolo J., Sol. Stat. Comm., 1994, 89, 393.

48. Stasyuk I.V., Czapla Z., Dacko S., Velychko O.V., J. Phys.: Condens. Matter, 2004, 161963.

49. Levitsky R.R., Zachek I.R., Vdovych A.S. Preprint of the Institute for Condensed Matter Physics, ICMP-09-02U, Lviv, 2009 (in Ukrainian).

50. Stasyuk I.V., Velychko O.V., Ferroelectrics, 2004, 300, 121.

\title{
Вплив поперечних електричних полів на діелектричні, п'єзоелектричні, пружні і теплові властивості сегнетової солі $\mathrm{NaKC}_{4} \mathrm{H}_{4} \mathrm{O}_{6} \cdot 4 \mathrm{H}_{2} \mathrm{O}$
}

\author{
Р.Р.Левицький ${ }^{1}$, І.Р.Зачек², А.С.Вдович ${ }^{1}$, І.В.Стасюк ${ }^{1}$ \\ ${ }^{1}$ Інститут фізики конденсованих систем НАН України вул. Свєнціцького, 1, Львів, 79011, Україна, \\ 2 Національний університет “Львівська політехніка”, вул. С. Бандери 12, 79013, Львів, Україна
}

Отримано 26 травня 2009 р.

Запропоновано модифіковану чотирипідграткову модель сегнетової солі шляхом врахування п'єзоелектричних взаємодій зі зсувними деформаціями $\varepsilon_{4}, \varepsilon_{5}$ i $\varepsilon_{6}$. В наближенні молекулярного поля отримано компоненти вектора поляризації та тензора статичної діелектричної проникності механічно затиснутого і вільного кристалів, їх п'єзоелектричні характеристики і пружні сталі. Вперше проведено ґрунтовне дослідження впливу поперечних полів на температури фазових переходів, діелектричні та пружні властивості сегнетової солі.

Ключові слова: сегнетоелектрики, сегнетова сіль, п'єзоелектричні модулі

PACS: 77.84.-s, 64.60.Cn, 77.22.-d, 77.80.-e, 77.80.Bh, 77.65.Bn 
